\title{
Vector aeroacoustics for a uniform mean flow: acoustic velocity and
}

\section{vortical velocity}

\author{
Yijun $\mathrm{Mao}^{1,2^{*}}$, Zhiwei $\mathrm{Hu}^{1 \dagger}$, Chen $\mathrm{Xu}^{1,2 \ddagger}$ \\ 1 University of Southampton, SO17 1BJ Southampton, United Kingdom \\ 2 Xi'an Jiaotong University, 710049 Xi'an, People's Republic of China \\ and \\ Ghader Ghorbaniasl ${ }^{3 \S}$ \\ 3 Vrije Universiteit Brussel (VUB), Pleinlaan 2, Brussels 1050, Belgium
}

Acoustic and vortical disturbances in uniform mean flow bounded with solid surfaces are investigated in this paper. A convective vector wave equation and a convection equation which describe, respectively, the acoustic velocity and vortical velocity in uniform mean flow are deduced by combining the Helmholtz-Hodge decomposition method with the method of Mao et al. (AIAA Journal, 2016, 54(6): 1922-1931). Analytical acoustic velocity integral formulations for the monopole and dipole sources in uniform mean flow are deduced from the developed vector wave equation, and are also verified through numerical test cases. Moreover, this paper clarifies that aerodynamic sound is radiated from the monopole source as well as the irrotational components of the dipole and quadrupole sources. The solenoidal parts of the dipole and quadrupole sources are acoustically non-radiating but they induce vortical disturbances in uniform mean flow.

\footnotetext{
Nomenclature

$c_{0} \quad=\quad$ speed of sound in fluid, $\mathrm{m} \mathrm{s}^{-1}$

$f \quad=\quad$ data surface function

$f_{0}=$ source pulsating frequency, $\mathrm{Hz}$
}

\footnotetext{
*Corresponding author. y.mao@ soton.ac.uk; maoyijun@mail.xjtu.edu.cn. Senior Member AIAA.

† Lecturer. z.hu@soton.ac.uk. Member AIAA.

*Research Fellow. c.xu@soton.ac.uk; xuchen1983@mail.xjtu.edu.cn.

§Professor. ghader.ghorbaniasl@vub.ac.be.
} 


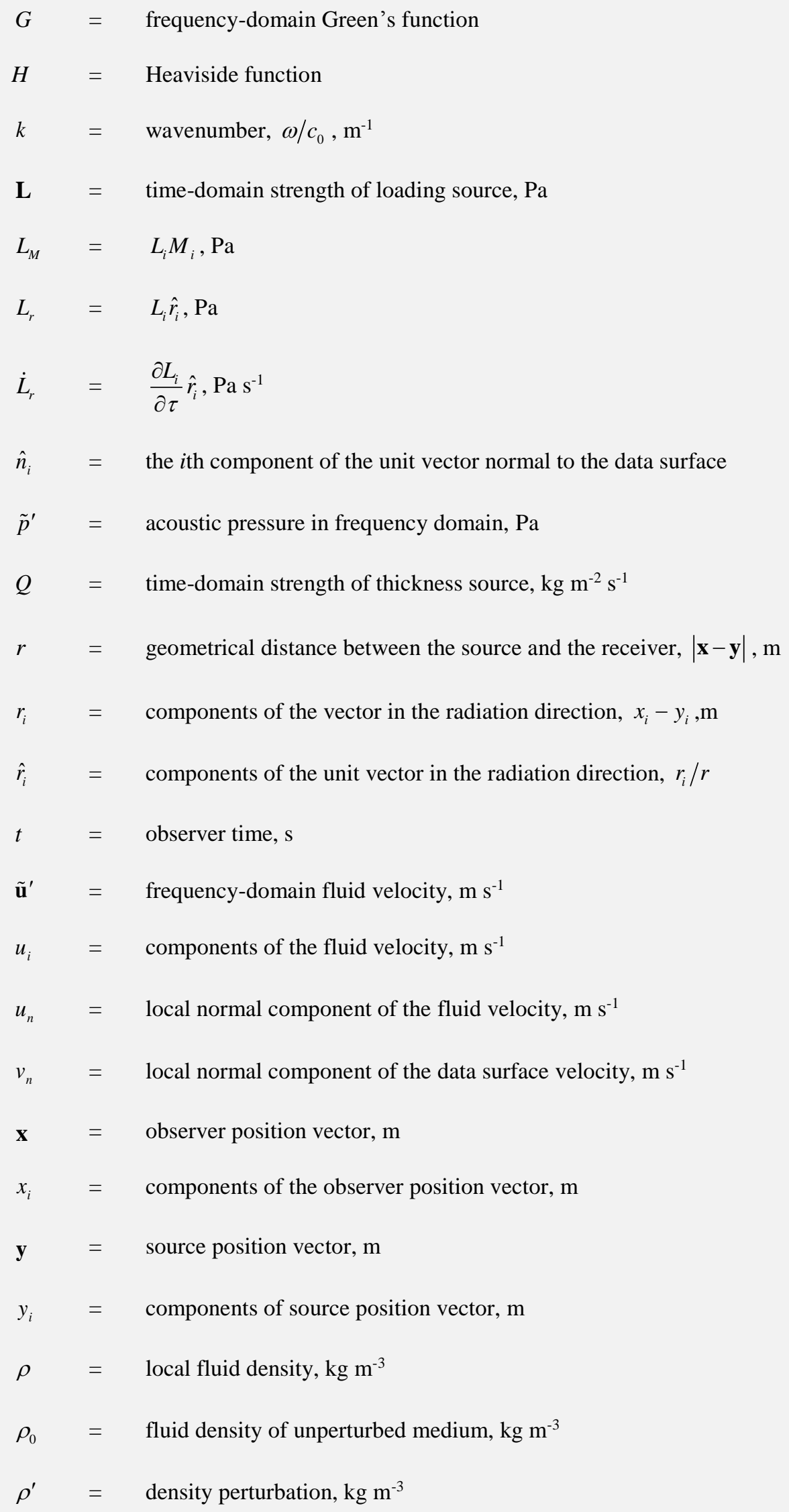




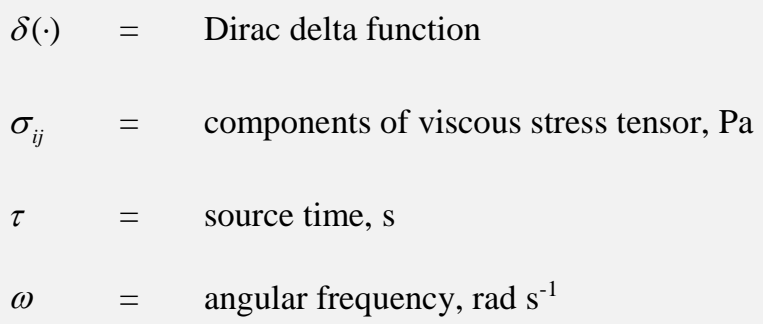




\section{Introduction}

$\mathrm{T}$

HE acoustic analogy proposed by Lighthill [1] initiates a systematic research on sound generated aerodynamically. Since then, various developments of the acoustic analogy have been carried out, for instances, to account for the effects of boundary conditions [2,3], initial conditions [4] and mean flow [5-8] on sound generation and propagation, and to understand the mechanism of aerodynamic noise by employing different variables for the wave operator and expressions of source terms $[7,9,10]$.

The above-mentioned investigations used a scalar, such as density or enthalpy, to describe the sound radiation from sources. The perturbation of acoustic scalars at any observer can be directly obtained from an integral computation using an appropriate Green's function [11-14]. These scalars establish a linear response of acoustic quantities, such as acoustic pressure and density perturbation, to aeroacoustic sources, but they are unable to provide information of detailed propagation path of the acoustic energy from sources to observers, which is important for designing acoustic treatments to absorb or insulate acoustic energy output from sources. Compared with acoustic scalars, acoustic vectors, such as acoustic intensity vector $[15,16]$ and acoustic energy streamline $[17,18]$, have an advantage in displaying the propagation path of the acoustic energy.

Recently, some investigations on aeroacoustic vectors have been carried out. Ghorbaniasl et al. [19] developed time-domain acoustic velocity formulations for monopole and dipole sources in arbitrary motion, and Mao et al. [20] deduced frequency-domain acoustic velocity formulations for rotating monopole and dipole sources with a constant angular speed. After that, acoustic intensity fields around sources [21] and scattering boundaries [22, 23] were visualized to illustrate the propagation of acoustic energy. All the above-mentioned investigations on the vector aeroacoustics method assume that acoustic medium is quiescent. This research aims to extend the vector aeroacoustics method to moving medium, which is meaningful in analyzing aeroacoustic problems in high-speed mean flows. Two types of potential engineering applications are listed below. Visualization of the acoustic intensity field around sources, such as airplanes, high-speed trains and rotors, is beneficial to reveal the propagation path of the acoustic energy. The acoustic impedance boundary condition, which directly correlates the acoustic pressure with the acoustic velocity, can be used to predict acoustic energy scattered by impedance surfaces immersed in moving fluids and to show primary acoustic-absorbing positions. 
As a first step, we consider the case of uniform mean flow in this paper, and earlier investigations in this area can be found in [24-28] among others. In order to analyze the effect of uniform mean flow on sound generation and propagation, Wells et al. [29] first established a convective Ffowcs Williams-Hawkings (FW-H) equation which contains modified aeroacoustic sources and a convective wave operator to describe sound generation and propagation, respectively. By starting from the convective FW-H equation, time-domain [30, 31] and frequencydomain [32] acoustic pressure integral formulations for the monopole and dipole sources in uniform mean flow have been deduced. The above-mentioned formulations explicitly account for the effect of the uniform mean flow on both the sound sources and sound propagation. On the other hand, the Lorentz or Prandtl-Glauert transformation [33, 34] is an alternative method to analyze sound propagation in uniform mean flow. Investigations have indicated that a moving background flow with high Mach numbers has a significant effect on sound generation and propagation [28, $30-32,35]$, implying that the classic exponent law of radiated acoustic power [1-3] is only valid approximately for low-Mach-number flows and should be corrected at high Mach numbers. In a quiescent acoustic medium, acoustic power can be computed once the distribution of the acoustic pressure is known on a closed far-field surface with an enough distance between sources and observers. However, the acoustic velocity is an essential physical parameter to compute the acoustic power output from sources in uniform mean flow [36]. To the best knowledge of the authors, analytical formulations of the acoustic pressure gradient for sources in uniform mean flow have only been derived recently [37], but no analytical acoustic velocity formulation is available in published literatures for sound radiated from aerodynamic sources in uniform mean flow.

From the viewpoint of general acoustics, in either the quiescent acoustic medium or the uniform mean flow, the acoustic velocity vector can be easily calculated from the gradient operation of the velocity potential. Indeed, the convective wave equation of the velocity potential is widely employed to analyze the effect of moving medium on sound propagation [38-41], and the acoustic pressure and acoustic velocity can be easily calculated from the velocity potential. However, it is difficult to derive a convective wave equation of aeroacoustics, in which the velocity potential is used as the acoustic variable on the left hand side (LHS) meanwhile aerodynamic sources are described on the right hand side (RHS).

In this paper, a convective vector wave equation and the corresponding acoustic velocity integral formulations are developed. Recently, based on the assumption of quiescent acoustic medium, Mao et al. [42] derived a vector wave equation of aeroacoustics by recasting the generalized continuity and momentum equations, which provides a 
method to derive the acoustic velocity formulations for sources in the quiescent acoustic medium. The paper extends the vector wave equation of aeroacoustics [42] to account for the effect of uniform mean flow. Since the velocity fluctuation simultaneously contains acoustic and vortical components in uniform mean flow without heat conduction [43], the Helmholtz-Hodge decomposition (HHD) is used to divide the acoustic and vortical velocity for deriving the convective vector wave equation. After that, the analytical acoustic velocity integral formulations for the monopole and dipole sources in uniform mean flow are deduced from the convective vector wave equation.

The remaining part of this paper is organized as follows. Section II derives a convective vector wave equation and a convection equation to describe, respectively, the acoustic and vortical disturbances in uniform mean flow bounded with solid surfaces. After that, analytical time-domain and frequency-domain acoustic velocity formulations for the monopole and dipole sources in uniform mean flow are deduced. In Section III, numerical test cases for stationary and rotating sources are carried out to verify the proposed acoustic velocity integral formulations, as well as to illustrate that the linearized Euler equation fails to describe the relationship between the acoustic pressure and the acoustic velocity for sound radiated from the dipole sources in uniform mean flow. Conclusions are drawn in Section IV.

\section{Convective vector wave equation and integral formulations}

\section{A. Helmholtz-Hodge decomposition}

The Helmholtz decomposition and HHD are fundamental theorems in fluid dynamics. Both decompositions state that a sufficiently smooth vector field can be decomposed into the sum of a longitudinal (diverging, curl-free, irrotational) vector field and a transverse (divergence-free, curling, rotational, solenoidal) vector field, that is

$$
\mathbf{\kappa}=\mathbf{\kappa}_{a}+\boldsymbol{\kappa}_{r}
$$

where $\boldsymbol{\kappa}$ is a vector, and subscripts $a$ and $r$ represent the irrotational and solenoidal components, respectively. The above decomposition guarantees the following identities:

$$
\nabla \times \mathbf{\kappa}_{a}=0 ; \quad \nabla \cdot \boldsymbol{\kappa}_{r}=0 ; \quad \nabla \times \mathbf{\kappa}=\nabla \times \boldsymbol{\kappa}_{r} ; \nabla \cdot \mathbf{\kappa}=\nabla \cdot \mathbf{\kappa}_{a}
$$

For a smooth unbounded fluid which approaches a uniform steady sate at infinity, the above decomposition is named the Helmholtz decomposition and this decomposition always exists, is orthogonal, and unique. On the other 
hand, if the fluid is bounded by solid surfaces, in order to ensure the decomposition is still unique, the following boundary conditions should be satisfied on the solid surfaces

$$
\mathbf{n} \times \boldsymbol{\kappa}_{a}=0 ; \quad \mathbf{n} \cdot \boldsymbol{\kappa}_{r}=0
$$

where $\mathbf{n}$ is the unit vector normal to the solid surfaces. The decomposition over the bounded fluid is usually named HHD. Details on these two decompositions can be found in [44-46].

By performing the above decomposition over the linearized Euler equation, we can deduce the following expression:

$$
\frac{\partial \mathbf{u}_{a}^{\prime}}{\partial t}+\frac{\partial \mathbf{u}_{r}^{\prime}}{\partial t}+\mathbf{U}_{\infty} \cdot \nabla \mathbf{u}_{a}^{\prime}+\mathbf{U}_{\infty} \cdot \nabla \mathbf{u}_{r}^{\prime}=-\nabla p^{\prime} / \rho_{0}
$$

where $\mathbf{u}_{a}^{\prime}$ and $\mathbf{u}_{r}^{\prime}$ are acoustic velocity and vortical velocity, respectively; $\mathbf{U}_{\infty}$ is the velocity of the uniform mean flow; $p^{\prime}$ is the pressure perturbation and $\rho_{0}$ is the undisturbed density. Additionally, for a second-order tensor, such as the Lighthill stress tensor $\mathbf{T}$, we have the following identities

$$
\nabla \cdot(\nabla \cdot \mathbf{T})_{r}=0 ; \quad \nabla \times(\nabla \cdot \mathbf{T})_{a}=0
$$

\section{B. Convective vector wave equation}

In order to consider the effect of uniform mean flow, we make the following decomposition of the local flow velocity $\mathbf{u}=\mathbf{U}_{\infty}+\mathbf{u}^{\prime}$, where $\mathbf{u}^{\prime}$ is the perturbed component of the local flow velocity. By using the above decomposition, generalized continuity and momentum equations which account for the uniform mean flow are expressed as follows [29-31]:

$$
\begin{gathered}
\frac{D[H(f) \rho]}{D t}+\frac{\partial\left[H(f) \rho u_{j}^{\prime}\right]}{\partial x_{j}}=Q^{M} \delta(f) \\
\frac{D\left[H(f) \rho u_{i}^{\prime}\right]}{D t}+\frac{\partial\left[H(f)\left(\rho u_{i}^{\prime} u_{j}^{\prime}+p \delta_{i j}-\sigma_{i j}\right)\right]}{\partial x_{j}}=L_{i}^{M} \delta(f)
\end{gathered}
$$

with

$$
\frac{D}{D t}=\frac{\partial}{\partial t}+U_{\infty i} \frac{\partial}{\partial x_{i}}
$$




$$
\begin{gathered}
Q^{M}=\rho_{0}\left(v_{n}-U_{\infty n}\right)+\rho\left[u_{n}^{\prime}-\left(v_{n}-U_{\infty n}\right)\right] \\
L_{i}^{M}=\left[\left(p-p_{0}\right) \delta_{i j}-\sigma_{i j}\right] \hat{n}_{j}+\rho u_{i}^{\prime}\left[u_{n}^{\prime}-\left(v_{n}-U_{\infty n}\right)\right]
\end{gathered}
$$

where superscript $M$ denotes source terms in uniform mean flow; $U_{\infty i}$ is the component of the uniform mean flow velocity in the $i^{\text {th }}$ direction and $U_{\infty n}=U_{\infty i} \hat{n}_{i} ; p$ is the static pressure; $\sigma_{i j}$ is the viscous stress tensor; $u_{i}$ is the $i^{\text {th }}$ component of fluid velocity; $u_{n}$ and $v_{n}$ are the normal components of the flow and data surface velocities, respectively; $\delta($.$) and H($.$) are the Dirac delta and Heaviside functions, respectively; \delta_{i j}$ is the Kronecker delta function; $f(\mathbf{x}, t)=0$ is either an impermeable solid surface or a permeable data surface in the flow region.

Since the static pressure $p_{0}$, density $\rho_{0}$ and sound speed $c_{0}$ are constant, the generalized momentum Eq. (7) can be expressed as

$$
\frac{D\left[H(f) \rho \mathbf{u}^{\prime}\right]}{D t}+\nabla \cdot\left[H(f) \mathbf{T}^{M}\right]+\nabla\left[H(f) \rho c_{0}^{2}\right]=\mathbf{L}^{M} \delta(f)
$$

with

$$
T_{i j}^{M}=\rho u_{i}^{\prime} u_{j}^{\prime}+\left[\left(p-p_{0}\right)-c_{0}^{2}\left(\rho-\rho_{0}\right)\right] \delta_{i j}-\sigma_{i j}
$$

Performing curl operation over Eq. (11) gives the following equation

$$
\nabla \times \frac{D\left[H(f) \rho \mathbf{u}^{\prime}\right]}{D t}=\nabla \times\left[\mathbf{L}^{M} \delta(f)\right]_{r}-\nabla \times\left[\nabla \cdot\left[H(f) \mathbf{T}^{M}\right]\right]_{r}
$$

The density perturbation $\rho^{\prime}$ is much smaller than the undisturbed density $\rho_{0}$ in the region outside the source $f>0$, thus we can have the following identity by using Eq. (2):

$$
\nabla \times\left[H(f) \rho \mathbf{u}^{\prime}\right]=\nabla \times\left[H(f) \rho_{0} \mathbf{u}^{\prime}\right]+\nabla \times\left[H(f) \rho^{\prime} \mathbf{u}^{\prime}\right]=\nabla \times\left[H(f) \rho_{0} \mathbf{u}_{r}^{\prime}\right]
$$

By substituting Eq. (14) into Eq. (13), we can obtain

$$
\nabla \times\left[\frac{D\left[H(f) \rho_{0} \mathbf{u}_{r}^{\prime}\right]}{D t}-\left[\mathbf{L}^{M} \delta(f)\right]_{r}+\left[\nabla \cdot\left[H(f) \mathbf{T}^{M}\right]\right]_{r}\right]=0
$$

In order to ensure that Eq. (15) is always valid, we must have the following identity 


$$
\frac{D\left[H(f) \rho_{0} \mathbf{u}_{r}^{\prime}\right]}{D t}-\left[\mathbf{L}^{M} \delta(f)\right]_{r}+\left[\nabla \cdot\left[H(f) \mathbf{T}^{M}\right]\right]_{r}=\mathbf{k}_{a} \text { or constant }
$$

where $\mathbf{k}_{a}$ is an irrotational quantity. However, all three terms on the LHS of Eq. (16) are solenoidal, thus their summation cannot be an irrotational quantity. Moreover, we study the vortical and acoustic disturbances at non-zero frequencies, and a constant only affects the component at zero frequency, thus Eq. (16) actually can be expressed by

$$
\frac{D\left[H(f) \mathbf{u}_{r}^{\prime}\right]}{D t}=\frac{\left[\mathbf{L}^{M} \delta(f)\right]_{r}}{\rho_{0}}-\frac{\left[\nabla \cdot\left[H(f) \mathbf{T}^{M}\right]\right]_{r}}{\rho_{0}}
$$

Eq. (17) indicates that the vortical velocity is originated from the solenoidal components of the dipole and quadrupole sources, and is convected in the uniform mean flow. Eq. (17) also implies that the monopole source has no contribution to the vortical velocity, thus the monopole source radiates only acoustic waves in uniform mean flow.

Now, the vector $\rho \mathbf{u}^{\prime}$ is employed as the variable of the wave operator to deduce the convective vector wave equation by following the method given in [42], but we emphasize that $\mathbf{u}^{\prime}$ is the total perturbated velocity rather than the acoustic velocity. A spatial derivative $\partial / \partial x_{i}$ of Eq. (6) is performed to obtain the following equation

$$
\frac{D}{D t} \frac{\partial[H(f) \rho]}{\partial x_{i}}+\frac{\partial^{2}\left[H(f) \rho u_{j}^{\prime}\right]}{\partial x_{i} \partial x_{j}}=\frac{\partial\left[Q^{M} \delta(f)\right]}{\partial x_{i}}
$$

Performing the material derivative $D / D t$ of Eq. (7) and multiplying it by a constant $1 / c_{0}^{2}$ yield

$$
\frac{1}{c_{0}^{2}} \frac{D^{2}\left[H(f) \rho u_{i}^{\prime}\right]}{D t^{2}}+\frac{1}{c_{0}^{2}} \frac{D}{D t} \frac{\partial\left[H(f)\left(\rho u_{i}^{\prime} u_{j}^{\prime}+p \delta_{i j}-\sigma_{i j}\right)\right]}{\partial x_{j}}=\frac{1}{c_{0}^{2}} \frac{D\left[L_{i}^{M} \delta(f)\right]}{D t}
$$

Subtracting Eq. (18) from Eq. (19) gives the following equation

$$
\begin{aligned}
\frac{1}{c_{0}^{2}} \frac{D^{2}\left[H(f) \rho u_{i}^{\prime}\right]}{D t^{2}}-\frac{\partial^{2}\left[H(f) \rho u_{j}^{\prime}\right]}{\partial x_{i} \partial x_{j}} & =-\frac{\partial\left[Q^{M} \delta(f)\right]}{\partial x_{i}}+\frac{1}{c_{0}^{2}} \frac{D\left[L_{i}^{M} \delta(f)\right]}{D t} \\
-\frac{1}{c_{0}^{2}} \frac{D}{D t} & {\left[\frac{\partial\left[H(f)\left(\rho u_{i}^{\prime} u_{j}^{\prime}+p \delta_{i j}-\left(\rho-\rho_{0}\right) c_{0}^{2} \delta_{i j}-\sigma_{i j}\right)\right]}{\partial x_{j}}\right] }
\end{aligned}
$$

Eq. (20) can also be equivalently expressed as

$$
\frac{1}{c_{0}^{2}} \frac{D^{2}\left(H(f) \rho \mathbf{u}^{\prime}\right)}{D t^{2}}-\nabla\left[\nabla \cdot\left(H(f) \rho \mathbf{u}^{\prime}\right)\right]=-\nabla\left(Q^{M} \delta(f)\right)+\frac{1}{c_{0}^{2}} \frac{D\left(\mathbf{L}^{M} \delta(f)\right)}{D t}-\frac{1}{c_{0}^{2}} \frac{D\left[\nabla \cdot\left(H(f) \mathbf{T}^{M}\right)\right]}{D t}
$$


The LHS of Eq. (21) is not a wave operator because the second term is not the Laplace operator. Applying the following identity

$$
\nabla\left[\nabla \cdot\left(H(f) \rho \mathbf{u}^{\prime}\right)\right]=\nabla^{2}\left[H(f) \rho \mathbf{u}^{\prime}\right]+\nabla \times\left[\nabla \times\left(H(f) \rho \mathbf{u}^{\prime}\right)\right]
$$

would turn the LHS of Eq. (21) into a wave operator with an additional source term $\nabla \times\left[\nabla \times\left(H(f) \rho \mathbf{u}^{\prime}\right)\right]$ on the RHS. An equivalent expression of the generalized momentum equation (7) is as follows:

$$
\frac{\partial\left[\rho u_{i}^{\prime} H(f)\right]}{\partial t}+U_{\infty j} \frac{\partial\left[\rho u_{i}^{\prime} H(f)\right]}{\partial x_{j}}+\frac{\partial\left[\left(\rho u_{i}^{\prime} u_{j}^{\prime}+p \delta_{i j}-\sigma_{i j}\right) H(f)\right]}{\partial x_{j}}=L_{i}^{M} \delta(f)
$$

Therefore, we can obtain the following expression from Eq. (23) by employing the identity of $\nabla \times(\nabla \kappa)=0$ where $\kappa$ is a scalar.

$$
\begin{aligned}
\nabla \times\left[\nabla \times\left(H(f) \rho \mathbf{u}^{\prime}\right)\right] & =\int_{0}^{t} \nabla \times\left[\nabla \times\left(\mathbf{L}^{M} \delta(f)\right)\right] \mathrm{d} t^{*} \\
& -\int_{0}^{t} \nabla \times\left\{\nabla \times\left[\nabla \cdot\left(\mathbf{T}^{M} H(f)\right)\right]\right\} \mathrm{d} t^{*} \\
& -\int_{0}^{t} \nabla \times\left\{\nabla \times\left[\mathbf{U}_{\infty} \cdot \nabla\left(H(f) \rho \mathbf{u}^{\prime}\right)\right]\right\} \mathrm{d} t^{*}
\end{aligned}
$$

Substituting Eqs. (22) and (24) into Eq. (21) gives

$$
\begin{aligned}
\frac{1}{c_{0}^{2}} \frac{D^{2}\left(H(f) \rho \mathbf{u}^{\prime}\right)}{D t^{2}}-\nabla^{2}\left(H(f) \rho \mathbf{u}^{\prime}\right)= & -\nabla\left(Q^{M} \delta(f)\right) \\
& +\frac{1}{c_{0}^{2}} \frac{D\left(\mathbf{L}^{M} \delta(f)\right)}{D t}+\int_{0}^{t} \nabla \times\left[\nabla \times\left(\mathbf{L}^{M} \delta(f)\right)\right] \mathrm{d} t^{*} \\
& -\frac{1}{c_{0}^{2}} \frac{D\left[\nabla \cdot\left(H(f) \mathbf{T}^{M}\right)\right]}{D t}-\int_{0}^{t} \nabla \times\left\{\nabla \times\left[\nabla \cdot\left(H(f) \mathbf{T}^{M}\right)\right]\right\} \mathrm{d} t^{*} \\
& -\int_{0}^{t} \nabla \times\left\{\nabla \times\left[\mathbf{U}_{\infty} \cdot \nabla\left(H(f) \rho \mathbf{u}^{\prime}\right)\right]\right\} \mathrm{d} t^{*}
\end{aligned}
$$

By using the following two identities

$$
\begin{gathered}
\int_{0}^{t} \nabla \times\left[\nabla \times\left(\mathbf{L}^{M} \delta(f)\right)\right] \mathrm{d} t^{*}=\int_{0}^{t} \nabla\left[\nabla \cdot\left(\mathbf{L}^{M} \delta(f)\right)\right] \mathrm{d} t^{*}-\int_{0}^{t} \nabla^{2}\left[\mathbf{L}^{M} \delta(f)\right] \mathrm{d} t^{*} \\
\int_{0}^{t} \nabla \times\left\{\nabla \times\left[\nabla \cdot\left(H(f) \mathbf{T}^{M}\right)\right]\right\} \mathrm{d} t^{*}=\int_{0}^{t} \nabla\left[\nabla \cdot\left[\nabla \cdot\left(H(f) \mathbf{T}^{M}\right)\right]\right] \mathrm{d} t^{*}-\int_{0}^{t} \nabla^{2}\left[\nabla \cdot\left(H(f) \mathbf{T}^{M}\right)\right] \mathrm{d} t^{*}
\end{gathered}
$$

Eq. (25) can be expressed as 


$$
\begin{aligned}
\frac{1}{c_{0}^{2}} \frac{D^{2}\left(H(f) \rho \mathbf{u}^{\prime}\right)}{D t^{2}}-\nabla^{2}\left(H(f) \rho \mathbf{u}^{\prime}\right)= & -\nabla\left(Q^{M} \delta(f)\right)+\int_{0}^{t} \nabla\left[\nabla \cdot\left(\mathbf{L}^{M} \delta(f)\right)\right] \mathrm{d} t^{*} \\
& -\int_{0}^{t} \nabla\left[\nabla \cdot\left[\nabla \cdot\left(H(f) \mathbf{T}^{M}\right)\right]\right] \mathrm{d} t^{*} \\
& +\frac{1}{c_{0}^{2}} \frac{D\left(\mathbf{L}^{M} \delta(f)\right)}{D t}-\int_{0}^{t} \nabla^{2}\left[\mathbf{L}^{M} \delta(f)\right] \mathrm{d} t^{*} \\
& -\frac{1}{c_{0}^{2}} \frac{D\left[\nabla \cdot\left(H(f) \mathbf{T}^{M}\right)\right]}{D t}+\int_{0}^{t} \nabla^{2}\left[\nabla \cdot\left(H(f) \mathbf{T}^{M}\right)\right] \mathrm{d} t^{*} \\
& -\int_{0}^{t} \nabla \times\left\{\nabla \times\left[\mathbf{U}_{\infty} \cdot \nabla\left(H(f) \rho \mathbf{u}^{\prime}\right)\right]\right\} \mathrm{d} t^{*}
\end{aligned}
$$

As shown in Appendix A, we can prove the following two identities:

$$
\begin{array}{r}
\frac{1}{c_{0}^{2}} \frac{D\left(\mathbf{L}^{M} \delta(f)\right)}{D t}-\int_{0}^{t} \nabla^{2}\left(\mathbf{L}^{M} \delta(f)\right) \mathrm{d} t^{*}= \\
-\frac{\mathbf{U}_{\infty} \cdot \nabla}{c_{0}^{2}}\left(\mathbf{L}^{M} \delta(f)\right) \\
-\frac{1}{c_{0}^{2}} \int_{0}^{t}\left(\mathbf{U}_{\infty} \cdot \nabla\right)\left(\mathbf{U}_{\infty} \cdot \nabla\right)\left(\mathbf{L}^{M} \delta(f)\right) \mathrm{d} t^{*} \\
\frac{1}{c_{0}^{2}} \frac{D\left[\nabla \cdot\left(H(f) \mathbf{T}^{M}\right)\right]}{D t}-\int_{0}^{t} \nabla^{2}\left[\nabla \cdot\left(H(f) \mathbf{T}^{M}\right)\right] \mathrm{d} t^{*}=-\frac{\mathbf{U}_{\infty} \cdot \nabla}{c_{0}^{2}}\left[\nabla \cdot\left(H(f) \mathbf{T}^{M}\right)\right] \\
-\frac{1}{c_{0}^{2}} \int_{0}^{t}\left(\mathbf{U}_{\infty} \cdot \nabla\right)\left(\mathbf{U}_{\infty} \cdot \nabla\right)\left[\nabla \cdot\left(H(f) \mathbf{T}^{M}\right)\right] \mathrm{d} t^{*}
\end{array}
$$

Thus, we can rewrite Eq. (28) as follows:

$$
\begin{aligned}
\frac{1}{c_{0}^{2}} \frac{D^{2}\left(H(f) \rho \mathbf{u}^{\prime}\right)}{D t^{2}}-\nabla^{2}\left(H(f) \rho \mathbf{u}^{\prime}\right) & =-\nabla\left(Q^{M} \delta(f)\right)+\int_{0}^{t} \nabla\left[\nabla \cdot\left(\mathbf{L}^{M} \delta(f)\right)\right] \mathrm{d} t^{*}-\int_{0}^{t} \nabla\left\{\nabla \cdot\left[\nabla \cdot\left(H(f) \mathbf{T}^{M}\right)\right]\right\} \mathrm{d} t^{*} \\
& -\frac{\mathbf{U}_{\infty} \cdot \nabla}{c_{0}^{2}}\left(\mathbf{L}^{M} \delta(f)\right)-\frac{1}{c_{0}^{2}} \int_{0}^{t}\left(\mathbf{U}_{\infty} \cdot \nabla\right)\left(\mathbf{U}_{\infty} \cdot \nabla\right)\left(\mathbf{L}^{M} \delta(f)\right) \mathrm{d} t^{*} \\
& +\frac{\mathbf{U}_{\infty} \cdot \nabla}{c_{0}^{2}}\left[\nabla \cdot\left(H(f) \mathbf{T}^{M}\right)\right]+\frac{1}{c_{0}^{2}} \int_{0}^{t}\left(\mathbf{U}_{\infty} \cdot \nabla\right)\left(\mathbf{U}_{\infty} \cdot \nabla\right)\left[\nabla \cdot\left(H(f) \mathbf{T}^{M}\right)\right] \mathrm{d} t^{*} \\
& -\int_{0}^{t} \nabla \times\left\{\nabla \times\left[\mathbf{U}_{\infty} \cdot \nabla\left(H(f) \rho \mathbf{u}^{\prime}\right)\right]\right\} \mathrm{d} t^{*}
\end{aligned}
$$

By employing the assumption of a small perturbation $\rho^{\prime}<<\rho_{0}$ and ignoring the second-order small quantity $\rho^{\prime} \mathbf{u}^{\prime}$ outside the source region, the convective vector wave equation (31) can be written as

$$
\begin{aligned}
\frac{1}{c_{0}^{2}} \frac{D^{2}\left(H(f) \rho_{0} \mathbf{u}^{\prime}\right)}{D t^{2}}-\nabla^{2}\left(H(f) \rho_{0} \mathbf{u}^{\prime}\right) & =-\nabla\left(Q^{M} \delta(f)\right)+\int_{0}^{t} \nabla\left[\nabla \cdot\left(\mathbf{L}^{M} \delta(f)\right)\right] \mathrm{d} t^{*}-\int_{0}^{t} \nabla\left\{\nabla \cdot\left[\nabla \cdot\left(H(f) \mathbf{T}^{M}\right)\right]\right\} \mathrm{d} t^{*} \\
& -\frac{\mathbf{U}_{\infty} \cdot \nabla}{c_{0}^{2}}\left(\mathbf{L}^{M} \delta(f)\right)-\frac{1}{c_{0}^{2}} \int_{0}^{t}\left(\mathbf{U}_{\infty} \cdot \nabla\right)\left(\mathbf{U}_{\infty} \cdot \nabla\right)\left(\mathbf{L}^{M} \delta(f)\right) \mathrm{d} t^{*} \\
& +\frac{\mathbf{U}_{\infty} \cdot \nabla}{c_{0}^{2}}\left[\nabla \cdot\left(H(f) \mathbf{T}^{M}\right)\right]+\frac{1}{c_{0}^{2}} \int_{0}^{t}\left(\mathbf{U}_{\infty} \cdot \nabla\right)\left(\mathbf{U}_{\infty} \cdot \nabla\right)\left[\nabla \cdot\left(H(f) \mathbf{T}^{M}\right)\right] \mathrm{d} t^{*} \\
& -\int_{0}^{t} \nabla \times\left\{\nabla \times\left[\mathbf{U}_{\infty} \cdot \nabla\left(H(f) \rho \mathbf{u}^{\prime}\right)\right]\right\} \mathrm{d} t^{*}
\end{aligned}
$$


The velocity fluctuation in the uniform mean flow usually contains both the acoustic and vortical components. Therefore, we perform the HHD over the velocity vector as well as the dipole and quadrupole sources. By performing divergence operation over Eq. (32) and by following the method to derive Eq. (17), we can obtain the following convective wave equation for the acoustic velocity

$$
\begin{aligned}
\frac{1}{c_{0}^{2}} \frac{D^{2}\left(H(f) \rho_{0} \mathbf{u}_{a}^{\prime}\right)}{D t^{2}}-\nabla^{2}\left(H(f) \rho_{0} \mathbf{u}_{a}^{\prime}\right) & =-\nabla\left(Q^{M} \delta(f)\right)+\int_{0}^{t} \nabla\left[\nabla \cdot[\mathbf{L} \delta(f)]_{a}\right] \mathrm{d} t^{*}-\int_{0}^{t} \nabla\left\{\nabla \cdot\left[\nabla \cdot\left(H(f) \mathbf{T}^{M}\right)\right]_{a}\right\} \mathrm{d} t^{*} \\
& -\frac{\mathbf{U}_{\infty} \cdot \nabla[\mathbf{L} \delta(f)]_{a}}{c_{0}^{2}}-\frac{1}{c_{0}^{2}} \int_{0}^{t}\left(\mathbf{U}_{\infty} \cdot \nabla\right)\left(\mathbf{U}_{\infty} \cdot \nabla\right)[\mathbf{L} \delta(f)]_{a} \mathrm{~d} t^{*} \\
& +\frac{\mathbf{U}_{\infty} \cdot \nabla}{c_{0}^{2}}\left[\nabla \cdot\left(H(f) \mathbf{T}^{M}\right)\right]_{a}+\frac{1}{c_{0}^{2}} \int_{0}^{t}\left(\mathbf{U}_{\infty} \cdot \nabla\right)\left(\mathbf{U}_{\infty} \cdot \nabla\right)\left[\nabla \cdot\left(H(f) \mathbf{T}^{M}\right)\right] \mathrm{d} t^{*}
\end{aligned}
$$

The last term on the RHS of Eq. (32) is a solenoidal vector, thus it has no contribution to the acoustic velocity and disappears in Eq. (33). Equation (33) is the vector wave equation describing acoustic velocity generation from aerodynamic sources and propagation in the uniform mean flow, where the sources $Q^{M}, \mathbf{L}^{M}$ and $\mathbf{T}^{M}$ are given earlier in Eqs. (9), (10) and (12), respectively. Especially, when the Mach number of uniform mean flow is zero, the last four terms on the RHS of Eq. (33) disappear, and Eq. (33) reduces to the vector wave equation given in [42].

\section{Acoustic velocity integral formulations for monopole and dipole sources}

Three-dimensional free space Green's function in time domain for the convective wave equation (33) is as follows [47]:

$$
g=\frac{\delta\left(t-\tau-R / c_{0}\right)}{4 \pi R^{*}}
$$

with

$$
\begin{aligned}
& R^{*}=r \sqrt{1 / \gamma^{2}+M_{\infty r}^{2}} \\
& R=\gamma^{2}\left(R^{*}-r M_{\infty r}\right)
\end{aligned}
$$

where $\gamma=\sqrt{1 /\left(1-M_{\infty}^{2}\right)}, M_{\infty r}=M_{\infty i} \hat{r}_{i}, \hat{r}_{i}=r_{i} / r, M_{\infty i}=U_{\infty i} / c_{0} ; r=|\mathbf{x}-\mathbf{y}|$ is the geometric distance between the source and the receiver; $R$ is the distance of sound wave travelling from the source to the observer, which is different from the geometric distance $r$ due to the convective effect of uniform mean flow. Note that this Green's function is only suitable for subsonic uniform mean flow because the factor $\gamma$ must be a real number [28]. 
By performing Fourier transformation, we can obtain the corresponding frequency-domain Green's function as follows:

$$
G(\mathbf{x}, \mathbf{y}, \omega)=\int_{-\infty}^{\infty} g(\mathbf{x}, \mathbf{y}, t-\tau) \mathrm{e}^{\mathrm{i} \omega(t-\tau)} \mathrm{d} t=\frac{\mathrm{e}^{\mathrm{i} k R}}{4 \pi R^{*}}
$$

It should be emphasized that Eq. (37) is valid for stationary sources as well as sources in a periodic motion, such as the source with a constant rotating speed [32], in which case both $R$ and $R^{*}$ are functions of the source time $\tau$. The first-order and second-order spatial derivatives of the frequency-domain Green's function are as follows:

$$
\begin{gathered}
\frac{\partial G(\mathbf{x}, \mathbf{y}, \omega)}{\partial x_{i}}=\frac{\mathrm{i} k \mathrm{e}^{\mathrm{i} k R} \hat{R}_{i}}{4 \pi R^{*}}-\frac{\mathrm{e}^{\mathrm{i} k R} \hat{R}_{i}^{*}}{4 \pi R^{* 2}}=\frac{\mathrm{e}^{\mathrm{i} k R}\left(\mathrm{i} k R^{*} \hat{R}_{i}-\hat{R}_{i}^{*}\right)}{4 \pi R^{* 2}} \\
\frac{\partial^{2} G(\mathbf{x}, \mathbf{y}, \omega)}{\partial x_{i} \partial x_{j}}=-\frac{k^{2} \mathrm{e}^{\mathrm{i} k R} \hat{R}_{i} \hat{R}_{j}}{4 \pi R^{*}}-\frac{\mathrm{i} k \mathrm{e}^{\mathrm{i} k R}\left(\hat{R}_{i}^{*} \hat{R}_{j}+\hat{R}_{i} \hat{R}_{j}^{*}+\gamma^{2} \hat{R}_{i}^{*} \hat{R}_{j}^{*}-\delta_{i j}-\gamma^{2} M_{\infty i} M_{\infty j}\right)}{4 \pi R^{* 2}}+\frac{\mathrm{e}^{\mathrm{i} k R}\left(3 \hat{R}_{i}^{*} \hat{R}_{j}^{*}-M_{\infty i} M_{\infty j}-\delta_{i j} / \gamma^{2}\right)}{4 \pi R^{* 3}}
\end{gathered}
$$

Similar to the FW-H equation, a permeable data surface could be used to reduce or even avoid the computational time consumed by the quadrupole volume source, therefore only the acoustic integral formulations for the monopole and dipole surface sources are derived in this paper. By starting from Eq. (33) and employing the convective Green's function (34), we can obtain the acoustic velocity formulation for the monopole source in uniform mean flow as follows:

$$
H(f) 4 \pi \rho_{0} u_{a, T i}^{\prime}(\mathbf{x}, t)=-\frac{\partial}{\partial x_{i}} \int_{-\infty}^{\infty} \int_{f>0} \frac{Q^{M} \delta(f) \delta\left(t-\tau-R / c_{0}\right)}{R^{*}} \mathrm{~d} \mathbf{y} \mathrm{d} \tau
$$

where subscript $i$ represents the component in the $i$ th direction. By employing the feature of the Dirac delta function to transfer the volume integral into the surface integral and to eliminate the temporal integral, Eq. (40) can be expressed as follows:

$$
H(f) 4 \pi \rho_{0} u_{a, T i}^{\prime}(\mathbf{x}, t)=-\frac{\partial}{\partial x_{i}} \int_{f=0}\left[\frac{Q^{M}}{R^{*}\left(1-M_{R}\right)}\right]_{\mathrm{ret}} \mathrm{d} S
$$

Equation (41) can be used to numerically compute the acoustic velocity, but it is computationally inefficient and is prone to numerical errors because of the spatial derivative calculation over the observer. By following the method of Farassat [48], the spatial derivative in Eq. (41) can be transferred into the temporal derivative, thus we can deduce the following integral equation without spatial derivative 


$$
H(f) 4 \pi \rho_{0} u_{a, T i}^{\prime}(\mathbf{x}, t)=\frac{1}{c_{0}} \frac{\partial}{\partial t} \int_{-\infty}^{\infty} \int_{f=0} \frac{Q^{M} \hat{R}_{i} \delta\left(t-\tau-R / c_{0}\right)}{R^{*}} \mathrm{~d} S \mathrm{~d} \tau+\int_{-\infty}^{\infty} \int_{f=0} \frac{Q^{M} \hat{R}_{i}^{*} \delta\left(t-\tau-R / c_{0}\right)}{R^{* 2}} \mathrm{~d} S \mathrm{~d} \tau
$$

Furthermore, we can obtain the following formulation by using the feature of the Dirac delta function to eliminate the temporal integral

$$
H(f) 4 \pi \rho_{0} u_{a, T i}^{\prime}(\mathbf{x}, t)=\frac{1}{c_{0}} \frac{\partial}{\partial t} \int_{f=0}\left[\frac{Q^{M} \hat{R}_{i}}{R^{*}\left(1-M_{R}\right)}\right]_{\mathrm{ret}} d S+\int_{f=0}\left[\frac{Q^{M} \hat{R}_{i}^{*}}{R^{* 2}\left(1-M_{R}\right)}\right]_{\mathrm{ret}} d S
$$

where subscript ret represents quantities inside the square brackets should be evaluated at the retarded time $\tau=t-R / c_{0}$. Equation (43) is an extension of the formulation V1 of Ghorbaniasl [19] for the monopole source, which avoids spatial derivation but still contains the derivative with respect to the observer time. The numerical differentiation can be employed to compute the time-domain acoustic velocity, and a fast Fourier transform can also be performed to directly compute the frequency-domain acoustic velocity.

Moreover, by following the derivation of formulation 1A of Farassat [48], an integral formulation eliminating both the temporal and spatial derivatives with respect to the observer can be deduced. However, the detailed derivation is long and we do not present the detailed expression in this paper. Note that the above time-domain formulation is only valid for sources in subsonic motion owing to the Doppler factor $1-M_{R}$ in the denominator. An improved formulation suitable for sources in supersonic motion can also be deduced by following the method of Farassat [48] to avoid the singularity caused by the Doppler term.

If the monopole source is in rotation with a constant angular speed or in other periodic motions, performing the Fourier transform on Eq. (42) and employing the frequency-domain Green function given in Eq. (37), give the following frequency-domain acoustic velocity integral formulation

$$
H(f) 4 \pi \rho_{0} \tilde{u}_{a, T i}^{\prime}(\mathbf{x}, \omega)=\int_{-\infty}^{\infty} \int_{f=0}\left[-\frac{\mathrm{i} k Q^{M} \hat{R}_{i}}{R^{*}}+\frac{Q^{M} \hat{R}_{i}^{*}}{R^{* 2}}\right] \mathrm{e}^{\mathrm{i} k R} \mathrm{e}^{\mathrm{i} \omega \tau} \mathrm{d} S \mathrm{~d} \tau
$$

where variables with a tilde $\sim$ denote the frequency-domain complex quantities.

Especially, when the source is stationary, we can obtain the following simplified time-domain formulation from Eq. (43)

$$
H(f) 4 \pi \rho_{0} u_{a, T i}^{\prime}(\mathbf{x}, t)=\frac{1}{c_{0}} \int_{f=0}\left[\frac{\dot{Q}^{M} \hat{R}_{i}}{R^{*}}\right]_{\mathrm{ret}} \mathrm{d} S+\int_{f=0}\left[\frac{Q^{M} \hat{R}_{i}^{*}}{R^{* 2}}\right]_{\mathrm{ret}} \mathrm{d} S
$$


The corresponding frequency-domain formulation can also be deduced as follows:

$$
H(f) 4 \pi \rho_{0} \tilde{u}_{a, T i}^{\prime}(\mathbf{x}, \omega)=-\int_{f=0} \frac{\mathrm{i} k \tilde{Q}^{M} \hat{R}_{i}}{R^{*}} \mathrm{e}^{\mathrm{i} k R} \mathrm{~d} S+\int_{f=0} \frac{\tilde{Q}^{M} \hat{R}_{i}^{*}}{R^{* 2}} \mathrm{e}^{\mathrm{i} k R} \mathrm{~d} S
$$

where the first and second terms on the RHS of Eq. (46) are, respectively, named the far-field and near-field terms owing to their different decaying rate on the radiation distance $R^{*}$. A numerical verification of the developed acoustic velocity formulations for the monopole source in uniform mean flow will be carried out in Section VI.

By starting from Eq. (33) and employing the convective Green's function (34), we can obtain the acoustic velocity formulation for the dipole source in uniform mean flow as follows:

$$
\begin{aligned}
H(f) 4 \pi \rho_{0} u_{a, L i}^{\prime}(\mathbf{x}, t)= & \int_{0}^{t} \frac{\partial^{2}}{\partial x_{i} \partial x_{j}} \int_{-\infty}^{\infty} \int_{f>0} \frac{L_{a, j}^{M} \delta\left(t-\tau-R / c_{0}\right)}{R^{*}} \delta(f) \mathrm{d} \mathbf{y d} \tau \mathrm{d} t^{*} \\
& -\frac{M_{\infty j}}{c_{0}} \frac{\partial}{\partial x_{j}} \int_{-\infty}^{\infty} \int_{f>0} \frac{L_{a, i}^{M} \delta\left(t-\tau-R / c_{0}\right)}{R^{*}} \delta(f) \mathrm{d} \mathbf{y} \mathrm{d} \tau \\
& -M_{\infty j} M_{\infty<} \frac{\partial^{2}}{\partial x_{j} \partial x_{k}} \int_{0}^{t} \int_{-\infty}^{\infty} \int_{f>0} \frac{L_{a, i}^{M} \delta\left(t-\tau-R / c_{0}\right)}{R^{*}} \delta(f) \mathrm{d} \mathbf{y d} \tau \mathrm{d} t^{*}
\end{aligned}
$$

The second and third terms on the RHS of Eq. (47) exist only in uniform mean flow. By performing the firstorder and second-order spatial derivatives over Eq. (47), we can deduce the following equation:

$$
\begin{aligned}
H(f) \rho_{0} u_{a, L i}^{\prime}(\mathbf{x}, t) & =\frac{\partial}{\partial t} \int_{-\infty}^{\infty} \int_{f>0} \frac{L_{a, R}^{M} \hat{R}_{i}+\left(M_{\infty R}-M_{\infty R}^{2}\right) L_{a, i}^{M}}{c_{0}^{2}} g \delta(f) \mathrm{d} \mathbf{y} \mathrm{d} \tau \\
& +\int_{-\infty}^{\infty} \int_{f>0} \frac{L_{a, R^{*}}^{M} \hat{R}_{i}+\left(L_{a, R}^{M}+\gamma^{2} L_{a, R^{*}}^{M}\right) \hat{R}_{i}^{*}-L_{a, i}^{M}}{c_{0} R^{*}} g \delta(f) \mathrm{d} \mathbf{y d} \tau \\
& +\int_{-\infty}^{\infty} \int_{f>0} \frac{\left(M_{\infty R^{*}}-2 M_{\infty R^{\prime}} M_{\infty R^{*}}+\gamma^{2} M_{\infty}^{2}-\gamma^{2} M_{\infty R^{*}}^{2}\right) L_{a, i}^{M}-\gamma^{2} L_{a, M_{\infty}}^{M} M_{\infty i}}{c_{0} R^{*}} g \delta(f) \mathrm{d} \mathbf{y} \mathrm{d} \tau \\
& +\int_{0}^{t} \int_{-\infty}^{\infty} \int_{f>0} \frac{3 L_{a, R^{*}}^{M} \hat{R}_{i}^{*}+\left(2 M_{\infty}^{2}-3 M_{\infty R^{*}}^{2}-1\right) L_{a, i}^{M}-L_{a, M_{\infty}}^{M} M_{\infty i}}{R^{2}} g \delta(f) \mathrm{d} \mathbf{y} \mathrm{d} \tau \mathrm{d} t^{*}
\end{aligned}
$$

The feature of the Dirac delta function is used to transfer volume integrals into surface integrals and to then eliminate the temporal integral, one can obtain the following expression 


$$
\begin{aligned}
H(f) 4 \pi \rho_{0} u_{a, L i}^{\prime}(\mathbf{x}, t)= & \frac{\partial}{\partial t} \int_{f=0}\left[\frac{L_{a, R}^{M} \hat{R}_{i}+\left(M_{\infty R}-M_{\infty R}^{2}\right) L_{a, i}^{M}}{c_{0}^{2} R^{*}\left(1-M_{R}\right)}\right]_{\mathrm{ret}} \mathrm{d} S \\
& +\int_{f=0}\left[\frac{L_{a, R^{*}}^{M} \hat{R}_{i}+\left(L_{a, R}^{M}+\gamma^{2} L_{a, R^{*}}^{M}\right) \hat{R}_{i}^{*}-L_{a, i}^{M}}{c_{0} R^{* 2}\left(1-M_{R}\right)}\right]_{\mathrm{ret}} \mathrm{d} S \\
& +\int_{f=0}\left[\frac{\left(M_{\infty R^{*}}-2 M_{\infty R^{\prime}} M_{\infty R^{*}}+\gamma^{2} M_{\infty}^{2}-\gamma^{2} M_{\infty R^{*}}^{2}\right) L_{a, i}^{M}-\gamma^{2} L_{a, M_{\infty}}^{M} M_{\infty}}{c_{0} R^{* 2}\left(1-M_{R}\right)}\right]_{\mathrm{ret}} \mathrm{d} S \\
& +\int_{0}^{t} \int_{f=0}\left[\frac{3 L_{a, R^{*}}^{M} \hat{R}_{i}^{*}+\left(2 M_{\infty}^{2}-3 M_{\infty R^{*}}^{2}-1\right) L_{a, i}^{M}-L_{a, M_{\infty}}^{M} M_{\infty i}}{R^{* 3}\left(1-M_{R}\right)}\right]_{\mathrm{ret}} \mathrm{d} S \mathrm{~d} t^{*}
\end{aligned}
$$

Equation (49) is just the extension of formulation V1 of Ghorbansial [19] for the dipole source. Formulation V1A for the dipole source in uniform mean flow can also be deduced by taking the temporal derivative of the first term on the RHS of Eq. (49). However, detailed derivation is very tedious, thus we do not present it in this paper.

For the rotating dipole source in uniform mean flow, performing the Fourier transform on Eq. (48) and employing the definition of the frequency-domain Green function, i.e., Eq. (37), give the following frequencydomain acoustic velocity integral formulation

$$
\begin{aligned}
H(f) 4 \pi \rho_{0} \tilde{u}_{a, L i}^{\prime}(\mathbf{x}, \omega)= & -\mathrm{i} k \int_{-\infty}^{\infty} \int_{f=0} \frac{L_{a, R}^{M} \hat{R}_{i}+\left(M_{\infty R}-M_{\infty R}^{2}\right) L_{a, i}^{M}}{c_{0} R^{*}} e^{\mathrm{i} k R} e^{\mathrm{i} \omega \tau} \mathrm{d} S \mathrm{~d} \tau \\
& +\int_{-\infty}^{\infty} \int_{f=0} \frac{L_{a, R^{*}}^{M} \hat{R}_{i}+\left(L_{a, R}^{M}+\gamma^{2} L_{a, R^{*}}^{M}\right) \hat{R}_{i}^{*}-L_{a, i}^{M}}{c_{0} R^{* 2}} e^{\mathrm{i} k R} e^{\mathrm{i} \omega \tau} \mathrm{d} S \mathrm{~d} \tau \\
& +\int_{-\infty}^{\infty} \int_{f=0} \frac{\left(M_{\infty R^{*}}-2 M_{\infty R} M_{\infty R^{*}}+\gamma^{2} M_{\infty}^{2}-\gamma^{2} M_{\infty R^{*}}^{2}\right) L_{a, i}^{M}-\gamma^{2} L_{a, M_{\infty}}^{M} M_{\infty i}}{c_{0} R^{* 2}} e^{\mathrm{i} k R} e^{\mathrm{i} \omega \tau} \mathrm{d} S \mathrm{~d} \tau \\
& +\frac{\mathrm{i}}{k} \int_{-\infty}^{\infty} \int_{f=0} \frac{3 L_{a, R^{*}}^{M} \hat{R}_{i}^{*}+\left(2 M_{\infty}^{2}-3 M_{\infty R^{*}}^{2}-1\right) L_{a, i}^{M}-L_{a, M_{\infty}}^{M} M_{\infty i}}{c_{0} R^{* 3} k R} e^{\mathrm{i} \omega \tau} \mathrm{d} S \mathrm{~d} \tau
\end{aligned}
$$

Especially, when the Mach number of uniform mean flow is equal to zero, Eq. (50) reduces to the formulation of FV1A given in [20]. When the source is stationary, Eq. (49) can be simplified to 


$$
\begin{aligned}
H(f) 4 \pi \rho_{0} \tilde{u}_{a, L i}^{\prime}(\mathbf{x}, t)= & \frac{\partial}{\partial t} \int_{f=0}\left[\frac{L_{a, R}^{M} \hat{R}_{i}+\left(M_{\infty R}-M_{\infty R}^{2}\right) L_{a, i}^{M}}{c_{0}^{2} R^{*}}\right]_{r e t} \mathrm{~d} S \\
& +\int_{f=0}\left[\frac{L_{a, R^{*}}^{M} \hat{R}_{i}+\left(L_{a, R}^{M}+\gamma^{2} L_{a, R^{*}}^{M}\right) \hat{R}_{i}^{*}-L_{a, i}^{M}}{c_{0} R^{* 2}}\right]_{r e t} \mathrm{~d} S \\
& +\int_{f=0}\left[\frac{\left(M_{\infty R^{*}}-2 M_{\infty R} M_{\infty R^{*}}+\gamma^{2} M_{\infty}^{2}-\gamma^{2} M_{\infty R^{*}}^{2}\right) L_{a, i}^{M}-\gamma^{2} L_{a, M_{\infty}}^{M} M_{\infty i}}{c_{0} R^{* 2}}\right] \mathrm{d} S \\
& +\int_{0}^{t} \int_{f=0}\left[\frac{3 L_{a, R^{*}}^{M} \hat{R}_{i}^{*}+\left(2 M_{\infty}^{2}-3 M_{\infty R^{*}}^{2}-1\right) L_{a, i}^{M}-L_{a, M_{\infty}}^{M} M_{\infty i}}{R^{* 3}}\right]_{r e t} \mathrm{~d} S \mathrm{~d} t^{*}
\end{aligned}
$$

The corresponding frequency-domain integral formulation is as follows:

$$
\begin{aligned}
H(f) 4 \pi \rho_{0} \tilde{u}_{a, L i}^{\prime}(\mathbf{x}, \omega)= & -\mathrm{i} k \int_{f=0} \frac{\tilde{L}_{a, R}^{M} \hat{R}_{i}+\left(M_{\infty}-M_{\infty R}^{2}\right) \tilde{L}_{a, i}^{M}}{c_{0} R^{*}} e^{\mathrm{i} k R} \mathrm{~d} S \\
& +\int_{f=0} \frac{\tilde{L}_{a, R^{*}}^{M} \hat{R}_{i}+\left(\tilde{L}_{a, R}^{M}+\gamma^{2} \tilde{L}_{a, R^{*}}^{M}\right) \hat{R}_{i}^{*}-\tilde{L}_{a, i}^{M}}{c_{0} R^{* 2}} e^{\mathrm{i} k R} \mathrm{~d} S \\
& +\int_{f=0} \frac{\left(M_{\infty R^{*}}-2 M_{\infty R} M_{\infty R^{*}}+\gamma^{2} M_{\infty}^{2}-\gamma^{2} M_{\infty R^{*}}^{2}\right) \tilde{L}_{a, i}^{M}-\gamma^{2} \tilde{L}_{a, M_{\infty}}^{M} M_{\infty i}}{c_{0} R^{* 2}} e^{\mathrm{i} k R} \mathrm{~d} S \\
& +\frac{\mathrm{i}}{k} \int_{f=0} \frac{3 \tilde{L}_{a, R^{*}}^{M} \hat{R}_{i}^{*}+\left(2 M_{\infty}^{2}-3 M_{\infty R^{*}}^{2}-1\right) \tilde{L}_{a, i}^{M}-\tilde{L}_{a, M_{\infty}}^{M} M_{\infty i}}{c_{0} R^{* 3}} e^{\mathrm{i} k R} \mathrm{~d} S
\end{aligned}
$$

\section{Numerical verification and discussions}

\section{A. Method of numerical verification}

In this section, numerical test cases are performed to verify the developed acoustic velocity formulations considering the effect of uniform mean flow. To the best knowledge of the authors, no benchmarking case has been published for the acoustic velocity related to sources in uniform mean flow. As shown in Eq. (17), the monopole source has no contribution to the vortical disturbance, thus the following linearized Euler equation expressed in frequency domain is used to verify the acoustic velocity formulations for the monopole source in uniform mean flow

$$
\mathrm{i} \omega \tilde{\mathbf{u}}^{\prime}-\mathbf{U}_{\infty} \cdot \nabla \tilde{\mathbf{u}}^{\prime}=\frac{\nabla \tilde{p}^{\prime}}{\rho_{0}}
$$


However, in order to filter the vortical velocity component stimulated from the dipole source, we should use the following frequency-domain expression obtained from Eq. (53) to verify the acoustic velocity formulation for the dipole source in uniform mean flow

$$
\mathrm{i} \omega\left(\nabla \cdot \tilde{\mathbf{u}}_{a}^{\prime}\right)-\nabla \cdot\left(\mathbf{U}_{\infty} \cdot \nabla \tilde{\mathbf{u}}_{a}^{\prime}\right)=\frac{\nabla^{2} \tilde{p}^{\prime}}{\rho_{0}}
$$

The terms on the RHS of the Eqs. (53) and (54) can be calculated with the acoustic pressure formulations proposed in [30-32]. A first-order discretization scheme is used to numerically compute the spatial derivatives related to the acoustic pressure and the acoustic velocity in Eqs. (53) and (54), thus we have the following two expressions:

$$
\begin{aligned}
& \underbrace{\mathrm{i} \omega \tilde{u}_{a, i}^{\prime}(\mathbf{x})-\sum_{j=1}^{3} \frac{U_{\infty j}\left[\tilde{u}_{a, i}^{\prime}\left(\mathbf{x}+\Delta l \mathbf{e}_{j}\right)-\tilde{u}_{a, i}^{\prime}(\mathbf{x})\right]}{\Delta l}}_{a_{i, L H S}}=\underbrace{\frac{1}{\rho_{0}} \frac{\tilde{p}^{\prime}\left(\mathbf{x}+\Delta l \mathbf{e}_{i}\right)-\tilde{p}^{\prime}(\mathbf{x})}{\Delta l}}_{a_{i, R H S}} \\
& \mathrm{i} \omega \sum_{i=1}^{3} \frac{\left[\tilde{u}_{a, i}^{\prime}\left(\mathbf{x}+\Delta l \mathbf{e}_{i}\right)-\tilde{u}_{a, i}^{\prime}(\mathbf{x})\right]}{\Delta l} \\
& \underbrace{-\sum_{i=1}^{3} \sum_{j=1}^{3} \frac{U_{\infty j}\left[\tilde{u}_{a, i}^{\prime}\left(\mathbf{x}+\Delta l \mathbf{e}_{i}+\Delta l \mathbf{e}_{j}\right)-\tilde{u}_{a, i}^{\prime}\left(\mathbf{x}+\Delta l \mathbf{e}_{i}\right)-\tilde{u}_{a, i}^{\prime}\left(\mathbf{x}+\Delta l \mathbf{e}_{j}\right)+\tilde{u}_{a, i}^{\prime}(\mathbf{x})\right]}{\Delta l^{2}}}_{b_{\text {LHS }}} \\
& =\underbrace{\frac{1}{\rho_{0}} \sum_{i=1}^{3} \frac{\tilde{p}^{\prime}\left(\mathbf{x}+\Delta l \mathbf{e}_{i}\right)-\tilde{p}^{\prime}\left(\mathbf{x}-\Delta l \mathbf{e}_{i}\right)+2 \tilde{p}^{\prime}(\mathbf{x})}{\Delta l^{2}}}_{b_{\text {RHS }}}
\end{aligned}
$$

where $\Delta l$ represents the distance between two stationary observers, and $\Delta l=10^{-6} \mathrm{~m}$ is used in this paper.

In all the numerical test cases presented in this Section, we make the following assumptions. The density and sound speed of the ambient flow are $\rho_{0}=1.2 \mathrm{~kg} / \mathrm{m}^{3}$ and $c_{0}=340 \mathrm{~m} / \mathrm{s}$. The Mach number of the uniform mean flow is selected as $\mathbf{M}_{\infty}=(0.3,0.4,0.5)$, ensuring that the uniform mean flow is subsonic. The pulsating frequency of the source is $f_{0}=100 \mathrm{~Hz}$.

For sound radiated from a stationary source in uniform mean flow, the source is located at the coordinate origin. 36 observers are evenly located on a circle in a plane at $z=10 \mathrm{~m}$ with a radius of $1 \mathrm{~m}$. The computational result outputs the directivity pattern to verify the developed acoustic velocity formulations. 
For sound radiated from a rotating source in uniform mean flow. The source rotates around the $z$-axis in the plane of $z=0$ with the radius of rotation of $0.8 \mathrm{~m}$, and the frequency of source rotation is $50 \mathrm{~Hz}$. Only one observer is located at $(1,1,10) \mathrm{m}$, and the acoustic velocity components in three directions are computed to verify the developed acoustic velocity formulations.

\section{B. Monopole point source in uniform mean flow}

The source strength of the monopole point source is $\int_{f=0} \tilde{Q}^{M} \mathrm{~d} S=0.01 \mathrm{~kg} / \mathrm{s}$. When the source is stationary, Eqs. (46) and the acoustic pressure formulation in [32] are used to compute the terms on the LHS and RHS of Eq. (55), respectively. Fig. 1 illustrates the components in three directions, where Re, Im and Abs represent the real part, imaginary part and the magnitude of the complex quantity, respectively. The computational results obtained from the two methods are consistent with each other, validating the developed acoustic velocity formulation for the stationary monopole source in uniform mean flow.

For the rotating monopole source, Eq. (44) and the acoustic pressure formulation [32] are employed to compute the terms on the LHS and RHS of Eq. (55), respectively. Fig. 2 displays the spectra obtained from the abovementioned two methods, and a good agreement between these two results verifies the frequency-domain acoustic velocity formulations for the rotating monopole source in uniform mean flow.

Moreover, in the test case of the rotating monopole source, we compute the acoustic velocity components with time-domain and frequency-domain formulations, respectively. In solving the time-domain formulation Eq. (43), the number of samples in one revolution for the rotating source is 360 ; the source-time dominant algorithm [49-51] is used to solve the retarded-time equation. The instantaneous acoustic velocity signals received by the stationary observer are linearly interpolated and then transferred into the frequency domain by performing a fast Fourier transform.

Fig. 3 compares the components of the acoustic velocity calculated with the time-domain and frequency-domain acoustic velocity formulations, in which TDNM and FDNM represent the time-domain numerical method and frequency-domain numerical method, respectively. The results obtained from different methods achieve a good agreement, further verifying that the proposed time-domain and frequency-domain acoustic velocity formulations can be used to accurately predict the acoustic velocity components of the sound radiated from the rotating monopole source in uniform mean flow. 


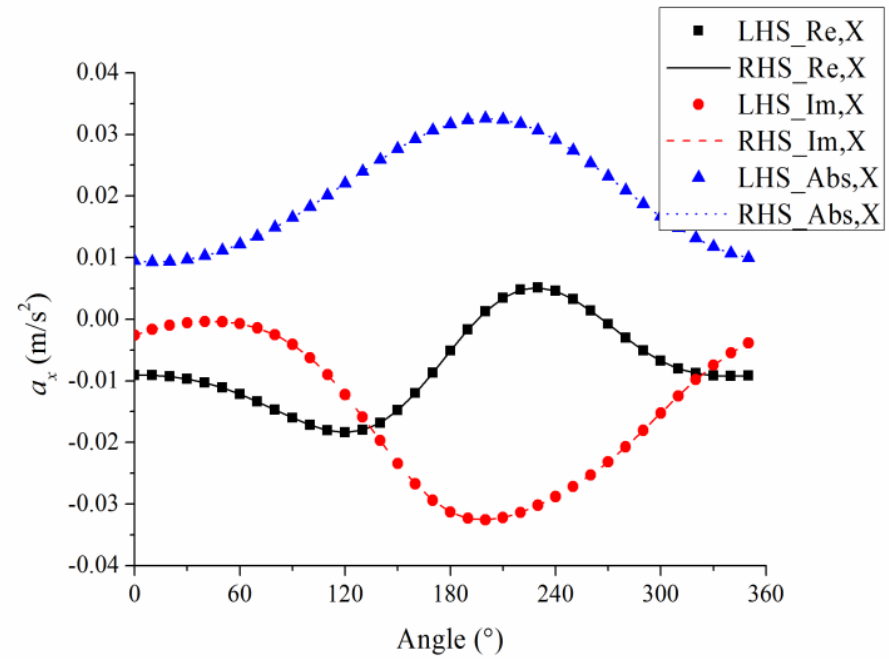

(a)

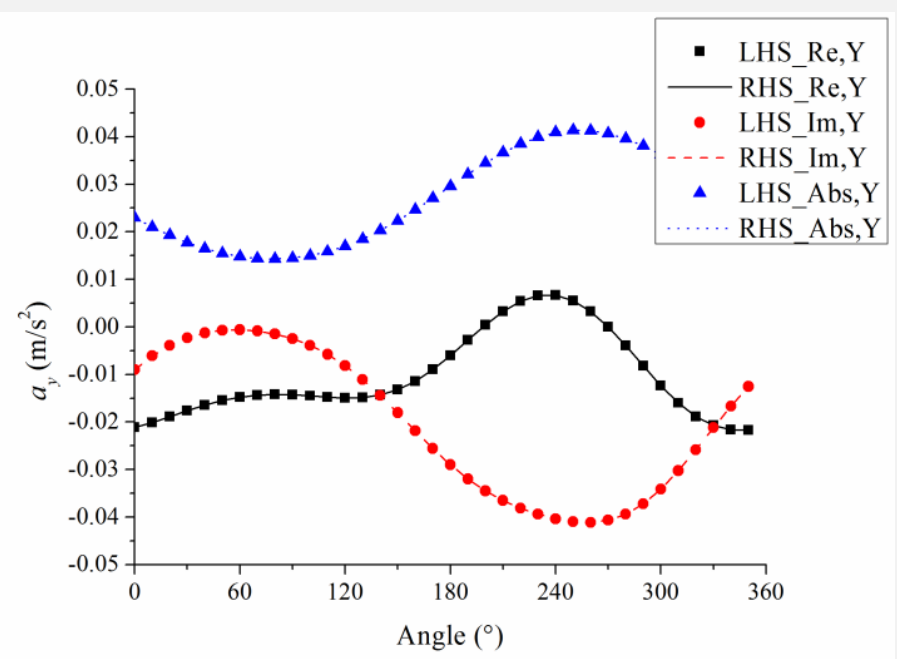

(b)

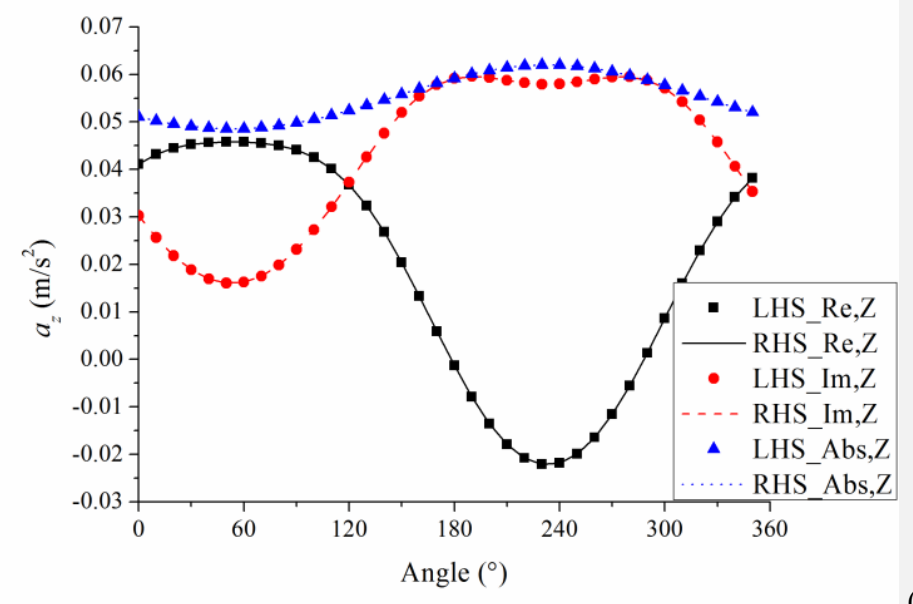

(c)

Fig. 1. Verification of acoustic velocity formulation for the stationary monopole source via Eq. (55): (a) $x$ component (b) $y$-component (c) $z$-component. 


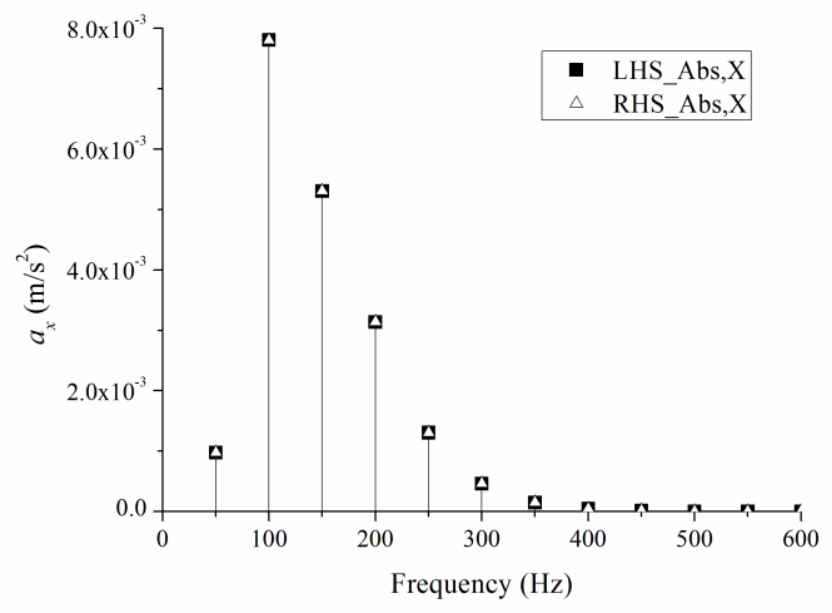

(a)

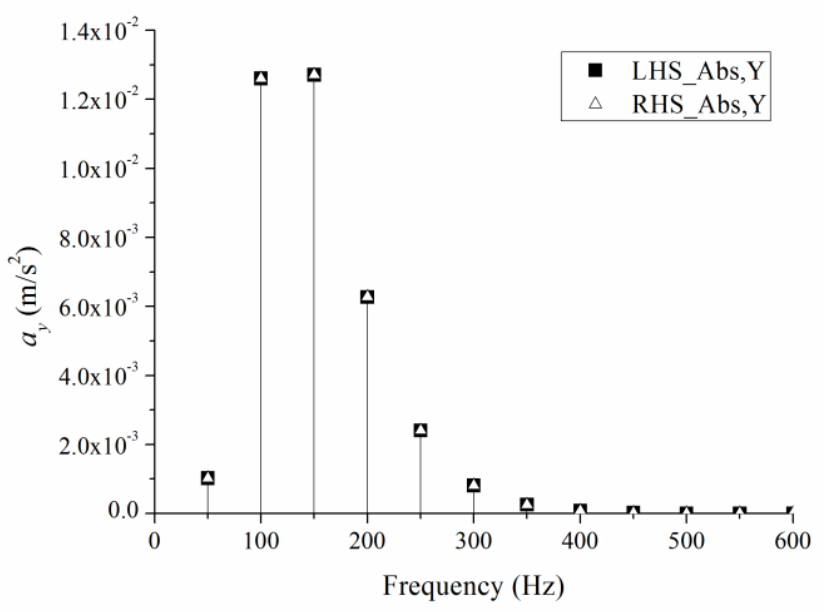

(b)

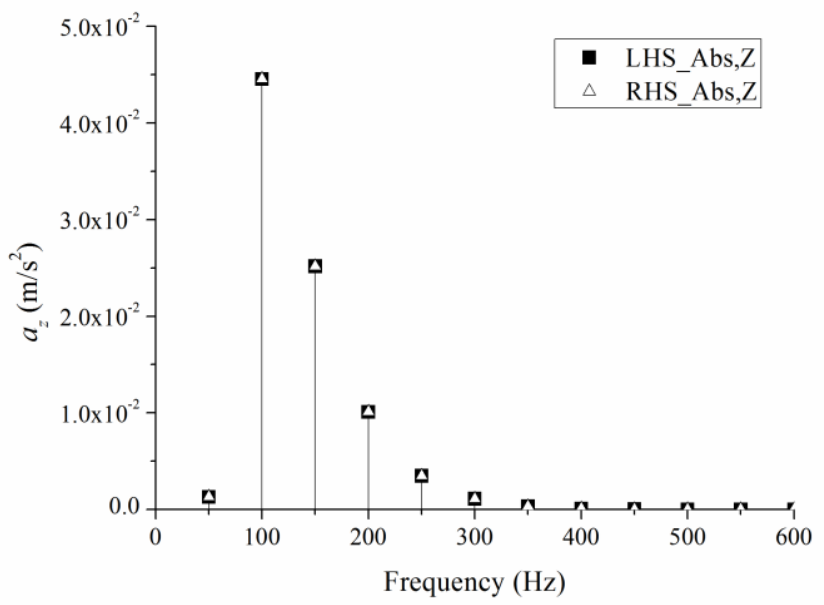

(c)

Fig. 2. Verification of acoustic velocity formulation for the rotating monopole source via Eq. (55): (a) magnitude of $a_{x}$; (b) magnitude of $a_{y}$; (c) magnitude of $a_{z}$ 


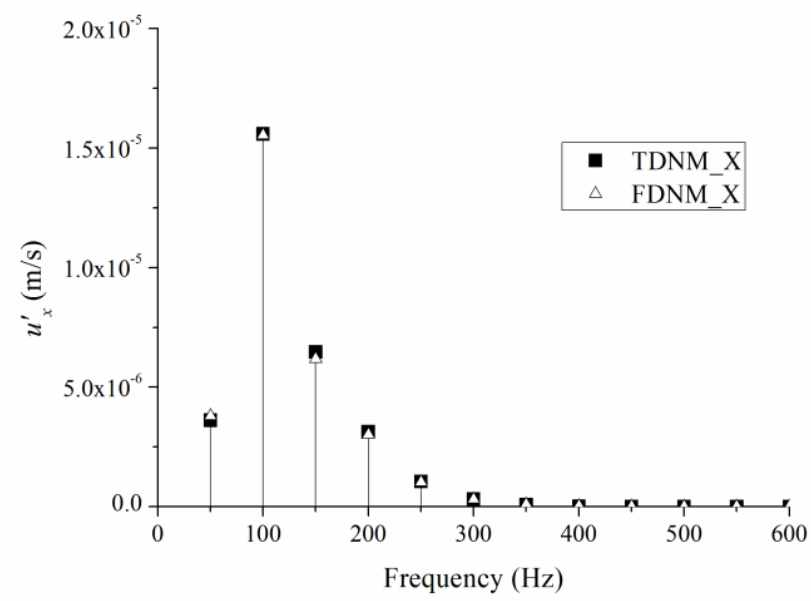

(a)

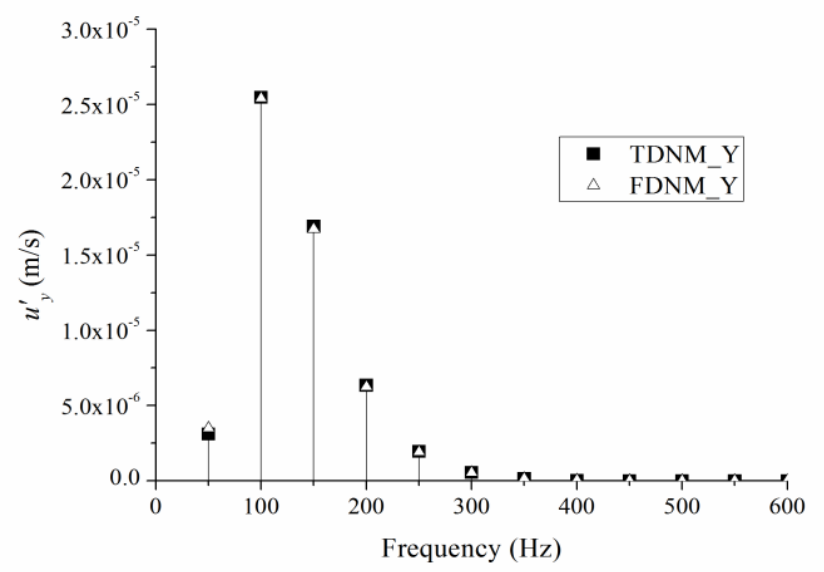

(b)

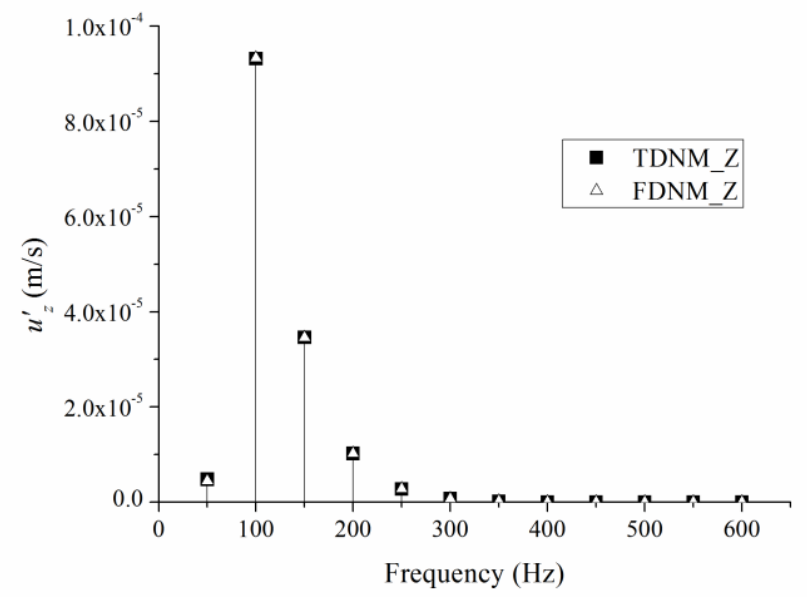

(c)

Fig. 3. Acoustic velocity components computed from the time-domain formulation (Eq. (43)) and frequencydomain formulation (Eq. (44)): (a) $x$-component (b) $y$-component (c) $z$-component. 


\section{Dipole point source in uniform mean flow}

For the stationary dipole point source, the irrotational components of source strength expressed in Cartesian coordinate system are given as $\int_{f=0} \tilde{L}_{a, i}^{M} \mathrm{~d} S=1 \mathrm{~N}(i=1,2,3)$. Equations (52) and the acoustic pressure formulation [32] are used to compute the terms on the LHS and RHS of Eq. (56), respectively. For the rotating dipole point source, the irrotational components of the rotating dipole source strength expressed in cylinder coordinate system are $\int_{f=0} \tilde{L}_{a, i}^{M} \mathrm{~d} S=1 \mathrm{~N}(i=R, \phi, Z)$. Equations (50) and the acoustic pressure formulation [32] are used to compute the terms on the LHS and RHS of Eq. (56), respectively.

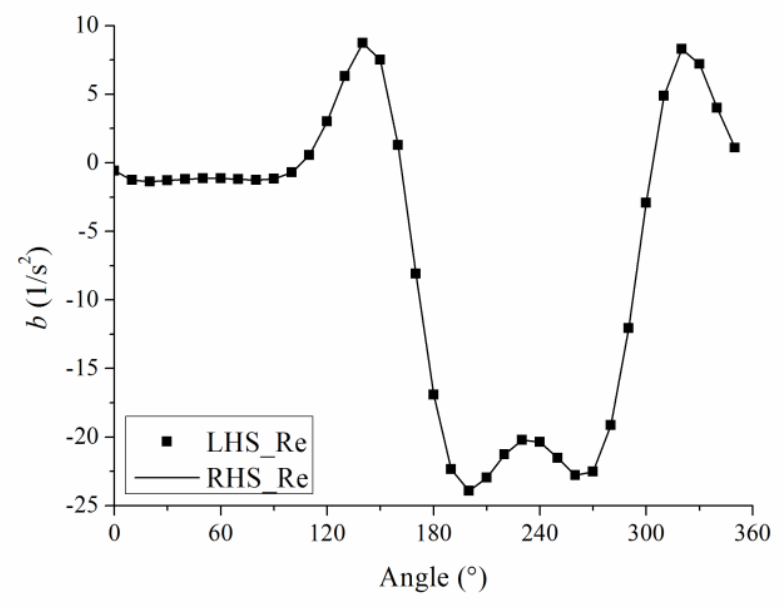

(a)

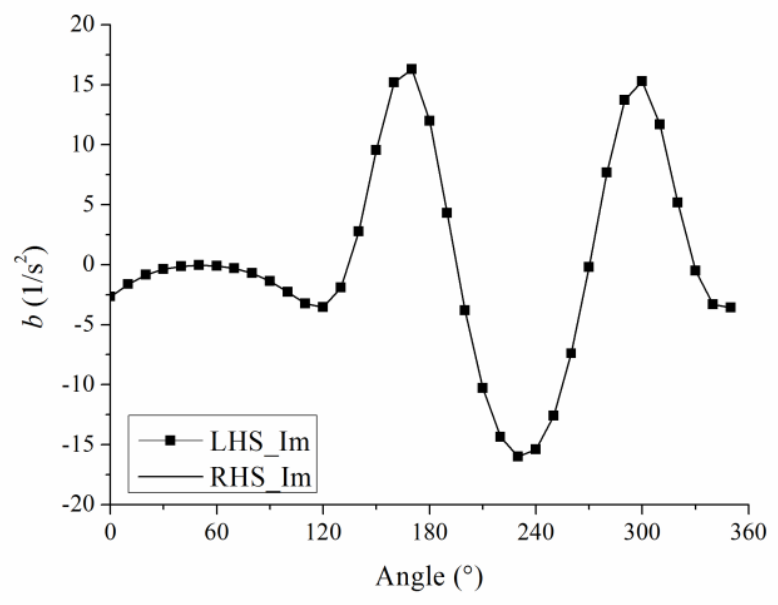

(b)

Fig. 4. Verification of acoustic velocity formulation for the stationary dipole source via Eq. (6.4): (a) real part; (b) imaginary part 
Since vortical disturbances can be stimulated by the dipole source in uniform mean flow, Eq. (56) is used to verify the developed acoustic velocity formulations for the dipole source. As shown in Fig. 4 and Fig. 5, a good agreement between the terms on the LHS and RHS for both the stationary and rotating cases verifies the acoustic velocity formulations for the dipole source in uniform mean flow. Moreover, Fig. 6 and Fig. 7 compare the computational results of the terms on the LHS and RHS of Eq. (55), we can find that obvious deviations always exist for both the stationary and rotating dipole sources, confirming that the linearized Euler equation cannot be used to calculate the acoustic velocity for the dipole source in uniform mean flow.

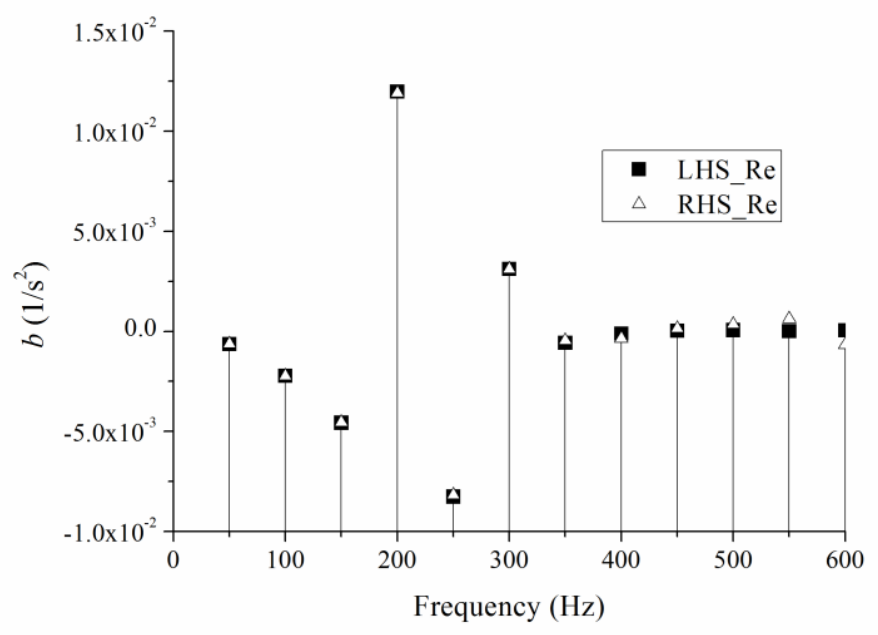

(a)

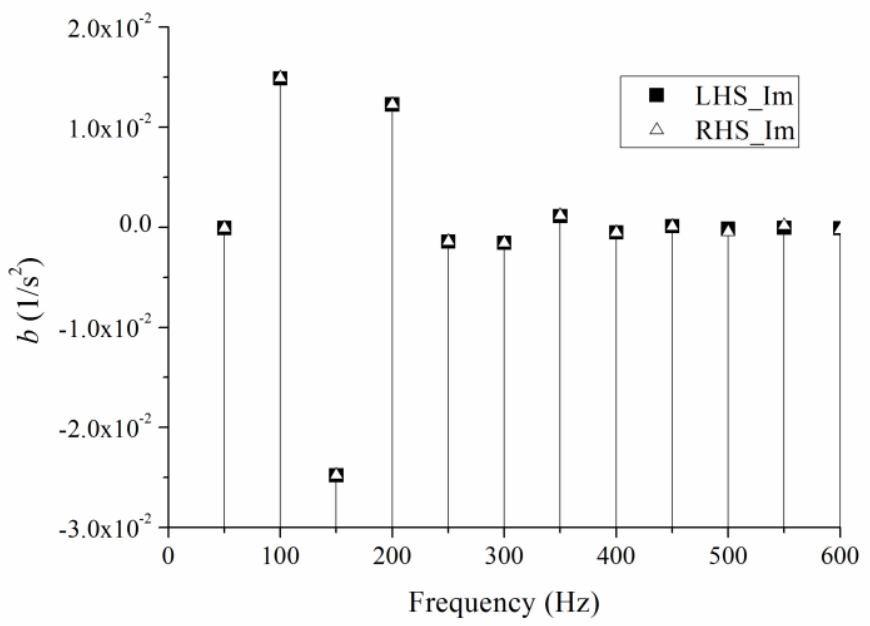

(b)

Fig. 5. Verification of acoustic velocity formulation for the rotating dipole source via Eq. (6.4): (a) real part; (b) imaginary part 


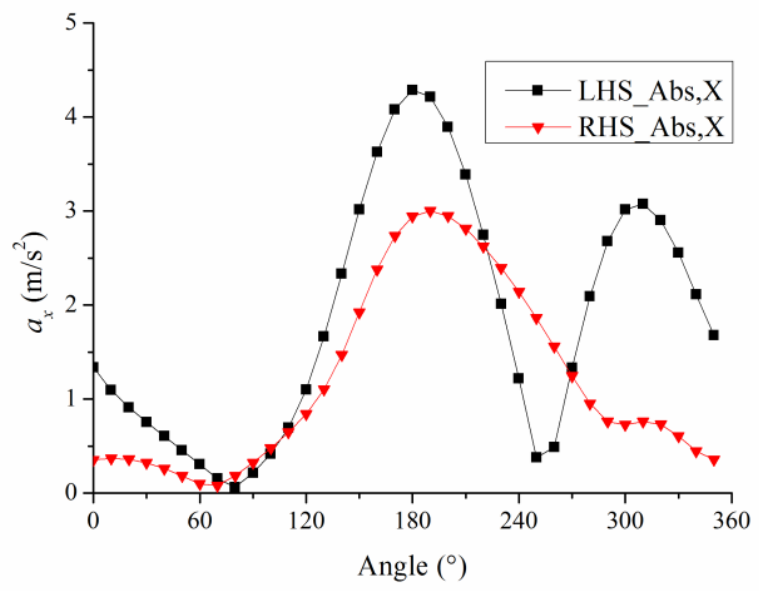

(a)

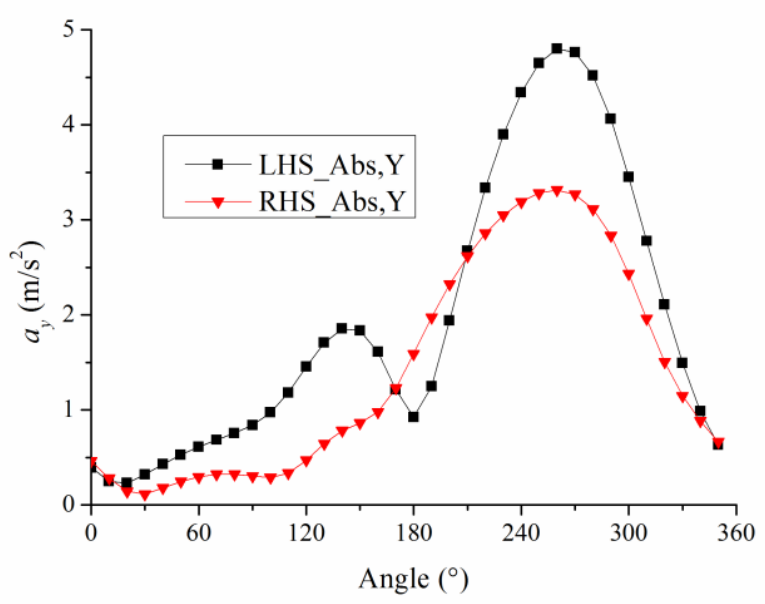

(b)

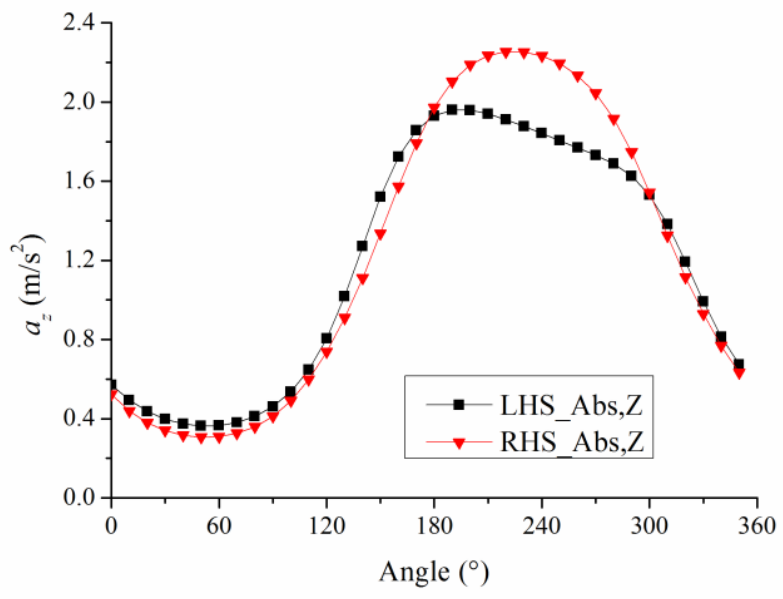

(c)

Fig. 6. Comparison of the terms on the two sides of Eq. (55) for the stationary dipole source: (a) $x$-component (b) $y$-component (c) $z$-component. 


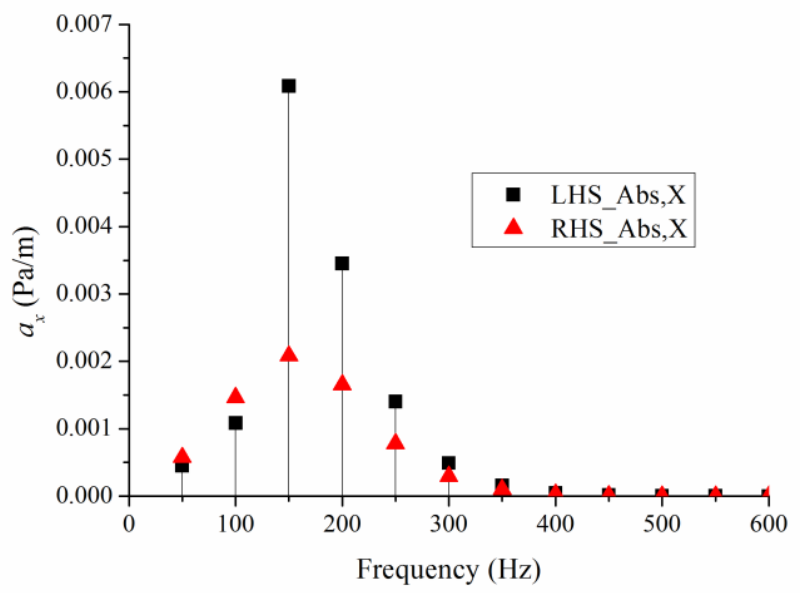

(a)

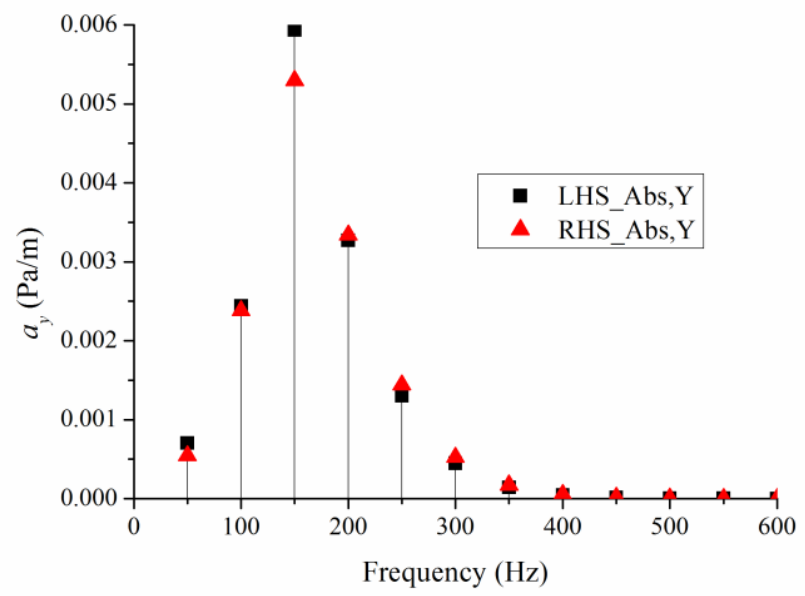

(b)

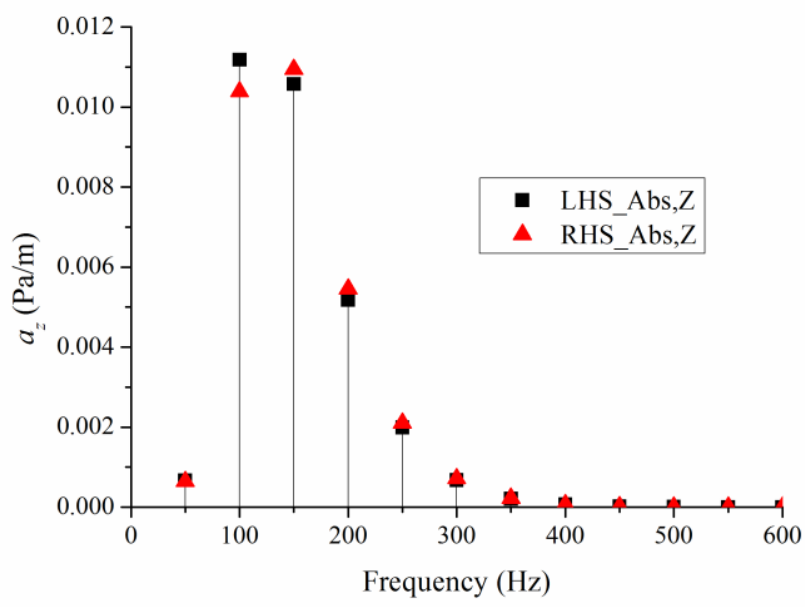

(c)

Fig. 7. Comparison of the terms on the two sides of Eq. (55) for the rotating dipole source: (a) $x$-component (b) $y$-component (c) z-component. 


\section{Conclusions}

The HHD can be used to decompose the flow velocity into irrotational and solenoidal components. By starting from the generalized compressible Navier-Stokes equations and by performing the HHD over the velocity vector as well as the dipole and quadrupole sources, a convective vector wave equation and a convection equation are deduced in this paper to describe, respectively, acoustic and vortical disturbances in uniform mean flow bounded with solid surfaces. The developed equations indicate that the monopole source as well as the irrotational parts of the dipole and quadrupole sources have contribution to sound radiation, and the solenoidal parts of the dipole and quadrupole sources stimulate vortical disturbances in uniform mean flow. Time-domain and frequency-domain acoustic velocity formulations for the monopole and dipole sources in uniform mean flow are deduced from the developed convective vector wave equation. Numerical test cases are performed to verify the developed acoustic velocity formulations for the monopole and dipole sources.

By employing the developed acoustic velocity formulations, we analyze the effect of the Mach number of uniform mean flow on the acoustic intensity field and acoustic power output in the next paper [52]. Moreover, the acoustic and vortical disturbances scattered by solid boundaries in uniform mean flow could be studied, and detailed investigations on this topic are planned in future research.

\section{Appendix A. Simplification of source expressions in the vector wave equation}

In uniform mean flow, we have the following identity by using the definition of the Green's function $g$

$$
\frac{1}{c_{0}^{2}} \frac{D^{2} g}{D t^{2}} \mathbf{\kappa}-\frac{\partial^{2} g}{\partial x_{j} \partial x_{j}} \mathbf{\kappa}=\mathbf{\kappa} \delta(\mathbf{x}-\mathbf{y}) \delta(t-\tau)
$$

where $\boldsymbol{\kappa}$ is an arbitrary vector. By performing the volume and temporal integrals over Eq. (A.1), we can obtain

$$
\frac{1}{c_{0}^{2}} \frac{D^{2}}{D t^{2}} \int_{-\infty}^{\infty} \int_{f>0} g \boldsymbol{\kappa} \mathrm{d} \mathbf{y d} \tau-\frac{\partial^{2}}{\partial x_{j} \partial x_{j}} \int_{-\infty}^{\infty} \int_{f>0} g \boldsymbol{\kappa} \mathrm{d} \mathbf{y} \mathrm{d} \tau=\int_{-\infty}^{\infty} \int_{f>0} \boldsymbol{\kappa} \delta(\mathbf{x}-\mathbf{y}) \delta(t-\tau) \mathrm{d} \mathbf{y} \mathrm{d} \tau
$$

When $\mathbf{x}$ does not coincide with $\mathbf{y}$ and $t$ does not coincide with $\tau$, the term on the RHS of Eq. (A.2) is equal to zero. Eq. (A.2) can be expressed as

$$
\frac{1}{c_{0}^{2}} \frac{D^{2}}{D t^{2}} \int_{-\infty}^{\infty} \int_{f>0} g \boldsymbol{\kappa} \mathrm{d} \mathbf{y} \mathrm{d} \tau=\frac{1}{c_{0}^{2}}\left(\frac{\partial}{\partial t}+\mathbf{U}_{\infty} \cdot \nabla\right) \frac{D}{D t} \int_{-\infty}^{\infty} \int_{f>0} g \boldsymbol{\kappa} \mathrm{d} \mathbf{y} \mathrm{d} \tau=\frac{\partial^{2}}{\partial x_{j} \partial x_{j}} \int_{-\infty}^{\infty} \int_{f>0} g \mathbf{K} \mathrm{d} \mathbf{y} \mathrm{d} \tau
$$


Performing temporal integral over Eq. (A.3) gives

$$
\begin{aligned}
& \frac{1}{c_{0}^{2}} \frac{D}{D t} \int_{-\infty}^{\infty} \int_{f>0} \boldsymbol{\kappa} g \mathrm{~d} \mathbf{y d} \tau-\frac{\partial^{2}}{\partial x_{j} \partial x_{j}} \int_{0}^{t} \int_{-\infty}^{\infty} \int_{f>0} \boldsymbol{\kappa} g \mathrm{~d} \mathbf{y d} \tau \mathrm{d} t^{*}=-\frac{1}{c_{0}^{2}} \int_{0}^{t} \frac{D}{D t} \int_{-\infty}^{\infty} \int_{f>0}\left(\mathbf{U}_{\infty} \cdot \nabla\right) \kappa g \mathrm{~d} \mathbf{y d} \tau \mathrm{d} t^{*} \\
& =-\frac{1}{c_{0}^{2}} \int_{-\infty}^{\infty} \int_{f>0}\left(\mathbf{U}_{\infty} \cdot \nabla\right) \kappa g \mathrm{~d} \mathbf{y d} \tau-\frac{1}{c_{0}^{2}} \int_{0}^{t} \int_{-\infty}^{\infty} \int_{f>0}\left(\mathbf{U}_{\infty} \cdot \nabla\right)\left(\mathbf{U}_{\infty} \cdot \nabla\right) \boldsymbol{\kappa} g \mathrm{~d} \mathbf{y d} \tau \mathrm{d} t^{*}
\end{aligned}
$$

If the vector $\boldsymbol{\kappa}$ is substituted by $\mathbf{L}^{M} \delta(f)$ and $\nabla \cdot\left[H(f) \mathbf{T}^{M}\right]$, respectively, we can obtain Eqs. (29) and (30).

\section{Appendix B. Alternative derivation of the acoustic velocity formulation for the monopole source in} uniform mean flow

The acoustic pressure formulation for a monopole source in uniform mean flow is as follows:

$$
p_{T}^{\prime}(\mathbf{x}, t)=\frac{D}{D t} \int_{-\infty}^{\infty} \int_{f>0} \frac{Q^{M} \delta(f) \delta\left(t-\tau-R / c_{0}\right)}{4 \pi R^{*}} \mathrm{~d} \mathbf{y} \mathrm{d} \tau
$$

Because the monopole source radiates only the acoustic wave rather than stimulates the vortical disturbance in uniform mean flow, thus the terms related to the vortical velocity in the linearized Euler equation (4) can be eliminated. Substituting Eq. (B.1) into Eq. (4), we can obtain the following expression

$$
\rho_{0} \frac{D \mathbf{u}_{a, T}^{\prime}(\mathbf{x}, t)}{D t}=-\nabla p_{T}^{\prime}(\mathbf{x}, t)=-\nabla\left[\frac{D}{D t} \int_{-\infty}^{\infty} \int_{f>0} \frac{Q^{M} \delta(f) \delta\left(t-\tau-R / c_{0}\right)}{4 \pi R^{*}} \mathrm{~d} \mathbf{y d} \tau\right]
$$

Furthermore, we can deduce the equation as follows:

$$
4 \pi \rho_{0} \mathbf{u}_{a, T}^{\prime}(\mathbf{x}, t)=-\nabla \int_{-\infty}^{\infty} \int_{f>0} \frac{Q^{M} \delta(f) \delta\left(t-\tau-R / c_{0}\right)}{R^{*}} \mathrm{~d} \mathbf{y} \mathrm{d} \tau
$$

Equation (B.3) is just the same as Eq. (40). We emphasize that this derivation is only suitable for the monopole source in uniform mean flow.

\section{Acknowledgments}

The research is supported by the National Natural Science Foundation of China (No. 51476123, No. 51511130075) and Newton Fund (No. IE141516). 


\section{References}

[1] Lighthill, M. J. "On Sound Generated Aerodynamically. I. General Theory," Proceedings of the Royal Society of London. Series A. Mathematical and Physical Sciences Vol. 211, No. 1107, 1952, pp. 564-587.

doi: $10.1098 /$ rspa.1952.0060

[2] Curle, N. "The Influence Of Solid Boundaries Upon Aerodynamic Sound," Proceedings of the Royal Society of London Series A. Mathematical And Physical Sciences Vol. 231, No. 1187, 1955, pp. 505-514.

doi: 10.1098/rspa.1955.0191

[3] Ffowcs Williams, J., and Hawkings, D. "Sound generation by turbulence and surfaces in arbitrary motion," Philosophical Transactions of the Royal Society of London Series A. Mathematical, Physical and Engineering Sciences Vol. 264, No. 1151,1969 , pp. 321-342.

doi: 10.1098/rsta.1969.0031

[4] Morfey, C. L., and Wright, M. C. M. "Extensions of Lighthill's acoustic analogy with application to computational aeroacoustics," Proceedings of the Royal Society of London. Series A. Mathematical and Physical Sciences Vol. 463, No. 2085, 2007, pp. 2101-2127.

doi: $10.1098 /$ rspa.2007.1864

[5] Goldstein, M. E. "An exact form of Lilley's equation with a velocity quadrupole/temperature dipole source term," Journal of Fluid Mechanics Vol. 443, 2001, pp. 231-236.

[6] Goldstein, M. E. "A generalized acoustic analogy," Journal of Fluid Mechanics Vol. 488, 2003, pp. 315-333. doi: $10.1017 / \mathrm{S} 0022112003004890$

[7] Lilley, G. "On the noise from jets," AGARD CP-131 Vol. 13, 1974, p. 12.

[8] Posson, H., and Peake, N. "The acoustic analogy in an annular duct with swirling mean flow," Journal of Fluid Mechanics Vol. 726, 2013, pp. 439-475.

doi: $10.1017 / J f m .2013 .210$

[9] Howe, M. "Contributions to the theory of aerodynamic sound, with application to excess jet noise and the theory of the flute," Journal of Fluid Mechanics Vol. 71, No. 04, 1975, pp. 625-673. doi: $10.1017 / \mathrm{S} 0022112075002777$

[10] Doak, P. E. "Fluctuating total enthalpy as a generalized acoustic field," Acoustical Physics Vol. 41, No. 5, 1995, pp. 677685.

[11] Farassat, F., and Succi, G. P. "A Review Of Propeller Discrete Frequency Noise Prediction Technology with Emphasis on two Current Methods for Time Domain Calculations," Journal of Sound and Vibration Vol. 71, No. 3, 1980, pp. 399-419. doi: 10.1016/0022-460x(80)90422-8 
[12] Farassat, F. "Linear Acoustic Formulas for Calculation Of Rotating Blade Noise," Aiaa Journal Vol. 19, No. 9, 1981, pp. 1122-1130. doi: $10.2514 / 3.60051$

[13] Brentner, K. S. "An efficient and robust method for predicting helicopter high-speed impulsive noise," Journal of Sound and Vibration Vol. 203, No. 1, 1997, pp. 87-100. doi: $10.1006 /$ jsvi.1996.0834

[14] Tang, H. , Qi, D. , and Mao, Y. "Analysis on the frequency-domain numerical method to compute the noise radiated from rotating sources," Journal Of Sound And Vibration Vol. 332, No. 23, 2013, pp. 6093-6103. doi: 10.1016/j.jsv.2013.06.020

[15] Fahy, F. Sound intensity. CRC Press, London, 2002.

[16] Jacobsen, F. "A note on instantaneous and time-averaged active and reactive sound intensity," Journal of Sound and Vibration Vol. 147, No. 3, 1991, pp. 489-496. doi: $10.1016 / 0022-460 X(91) 90496-7$

[17] Waterhouse, R. V., Yates, T. W., Feit, D., and Liu, Y. N. "Energy Streamlines Of a Sound Source," Journal of the Acoustical Society of America Vol. 78, No. 2, 1985, pp. 758-762. doi: $10.1121 / 1.392445$

[18] Waterhouse, R. V., and Feit, D. "Equal-Energy Streamlines," Journal of the Acoustical Society of America Vol. 80, No. 2, 1986, pp. 681-684. doi: 10.1121/1.394064

[19] Ghorbaniasl, G., Carley, M., and Lacor, C. "Acoustic velocity formulation for sources in arbitrary motion," Aiaa Journal Vol. 51, No. 3, 2013, pp. 632-642. doi: 10.2514/1.J051958

[20] Mao, Y., Zhang, Q., Xu, C., and Qi, D. "Two types of frequency-domain acoustic-velocity formulations for rotating thickness and loading sources," AIAA Journal Vol. 53, No. 3, 2015, pp. 713-722. doi: $10.2514 / 1 . j 053230$

[21] Mao, Y. , Xu, C., and Qi, D. "Computation of instantaneous and time-averaged active acoustic intensity field around rotating source," Journal of Sound and Vibration Vol. 337, 2015, pp. 95-115. doi: 10.1016/j.jsv.2014.10.023

[22] Mao, Y., Cai, J. , Gu, Y. , and Qi, D. "Direct evaluation of acoustic intensity vector field around impedance scattering body," AIAA Journal Vol. 53, No. 5, 2015, pp. 1362-1371. doi: 10.2514/1.J053431 
[23] Mao, Y., Gu, Y., and Xu, C. "Validation of Frequency-domain method to compute noise radiated from rotating source and scattered by surface," AIAA Journal Vol. 54, No. 4, 2016, pp. 1188-1197.

doi: 10.2514/1.J053674

[24] Lighthill, M. "The fourth annual fairey lecture: the propagation of sound through moving fluids," Journal of Sound and Vibration Vol. 24, No. 4, 1972, pp. 471-492. doi: $10.1016 / 0022-460 X(72) 90718-3$

[25] Dowling, A. "Convective amplification of real simple sources," Journal of Fluid Mechanics Vol. 74, No. 03, 1976, pp. 529546. doi: $10.1017 / \mathrm{S} 0022112076001936$

[26] Levine, H. "A Note on Sound Radiation into a Uniformly Flowing Medium," Journal of Sound and Vibration Vol. 71, No. 1, 1980, pp. $1-8$. doi: 10.1016/0022-460x(80)90403-4

[27] Lakhtakia, A., Varadan, V. K., and Varadan, V. V. "Green's functions for propagation of sound in a simply moving fluid," The Journal of the Acoustical Society of America Vol. 85, No. 5, 1989, pp. 1852-1856. doi: $10.1121 / 1.397892$

[28] Chapman, C. J. "Similarity variables for sound radiation in a uniform flow," Journal of Sound and Vibration Vol. 233, No. 1, 2000, pp. 157-164. doi: 10.1006/jsvi.1999.2800

[29] Wells, V. L., and Han, A. Y. "Acoustics Of a Moving Source In a Moving Medium with Application To Propeller Noise," Journal of Sound and Vibration Vol. 184, No. 4, 1995, pp. 651-663. doi: $10.1006 /$ jsvi.1995.0339

[30] Najafi-Yazdi, A., Bres, G. A., and Mongeau, L. "An acoustic analogy formulation for moving sources in uniformly moving media," Proceedings of the Royal Society Series A. Mathematical Physical and Engineering Sciences Vol. 467, No. 2125, 2011, pp. 144-165. doi: $10.1098 /$ rspa.2010.0172

[31] Ghorbaniasl, G., and Lacor, C. "A moving medium formulation for prediction of propeller noise at incidence," Journal of Sound and Vibration Vol. 331, No. 1, 2012, pp. 117-137. doi: 10.1016/j.jsv.2011.08.018

[32] Xu, C., Mao, Y., and Qi, D. "Frequency-domain acoustic pressure formulation for rotating source in uniform subsonic inflow with arbitrary direction," Journal of Sound and Vibration Vol. 333, 2014, pp. 3081-3091. doi: 10.1016/j.jsv.2014.03.004 
[33] Gregory, A. L., Sinayoko, S., Agarwal, A., and Lasenby, J. "An acoustic space-time and the Lorentz transformation in aeroacoustics," International Journal of Aeroacoustics Vol. 14, No. 7, 977-1003, 2015. doi: 10.1260/1475-472X.14.7.977

[34] Rienstra, S. W., and Hirschberg, A. "An introduction to acoustics." 2013.

[35] Mao, Y., Xu, C., and Qi, D. "Analytical solution for sound radiated from the rotating point source in uniform subsonic axial flow," Applied Acoustics Vol. 92, 2015, pp. 6-11.

doi: 10.1016/j.apacoust.2014.12.012

[36] Goldstein, M. E. Aeroacoustics. New York: McGraw-Hill International Book Company, 1976.

[37] Ghorbaniasl, G., Huang, Z., Siozos-Rousoulis, L., and Lacor, C. "Analytical acoustic pressure gradient prediction for moving medium problems," Proceedings of the Royal Society of London Series A. Mathematical and Physical Sciences Vol. 471, 2015, p. 20150342.

[38] Lee, Y. W., and Lee, D. J. "Derivation and implementation of the boundary integral formula for the convective acoustic wave equation in time domain," The Journal of the Acoustical Society of America Vol. 136, No. 6, 2014, pp. $2959-2967$. doi:10.1121/1.4898427

[39] Astley, R., and Bain, J. "A three-dimensional boundary element scheme for acoustic radiation in low Mach number flows," Journal of sound and vibration Vol. 109, No. 3, 1986, pp. 445-465. doi: 10.1016/S0022-460X(86)80381-9

[40] Wu, T., and Lee, L. "A direct boundary integral formulation for acoustic radiation in a subsonic uniform flow," Journal of sound and vibration Vol. 175, No. 1, 1994, pp. 51-63.

doi: $10.1006 /$ jsvi.1994.1310

[41] De Lacerda, L., Wrobel, L., and Mansur, W. "A boundary integral formulation for two - dimensional acoustic radiation in a subsonic uniform flow," The Journal of the acoustical society of America Vol. 100, No. 1, 1996, pp. 98-107. doi: $10.1121 / 1.415871$

[42] Mao, Y., Tang, H., and Xu, C. "Vector wave equation of aeroacoustics and acoustic velocity formulations for quadrupole source," AIAA Journal Vol. 54, No. 6, 2016, pp. 1922-1931. doi: 10.2514/1.J054687

[43] Kovasznay, L. S. G. "Turbulence in Supersonic Flow," Journal of the Aeronautical Sciences Vol. 20, No. 10, 1953, pp. 657-674.

doi: $10.2514 / 8.2793$

[44] Chorin, A. J., and Marsden, J. E. A mathematical introduction to fluid mechanics: Springer, 2000. 
[45] Denaro, F. M. "On the application of the Helmholtz-Hodge decomposition in projection methods for incompressible flows with general boundary conditions," International Journal for Numerical Methods in Fluids Vol. 43, No. 1, 2003 , pp. 43-69. doi: $10.1002 /$ fld. 598

[46] Bhatia, H., Norgard, G., Pascucci, V., and Bremer, P. T. "The Helmholtz-Hodge decomposition-a survey," IEEE Transactions on visualization and computer graphics Vol. 19, No. 8, 2013, pp. 1386-1404.

[47] Carley, M., Fitzpatrick. "Linear acoustic formulae for calculation of rotating blade noise with asymmetric inflow." AIAA Paper 96-1789.

[48] Farassat, F. "Derivation of Formulations 1 and 1A of Farassat." NASA/TM-2007-214853. 2007.

[49] Brentner, K. S., and Farassat, F. "Modeling aerodynamically generated sound of helicopter rotors," Progress in Aerospace Sciences Vol. 39, No. 2-3, 2003, pp. 83-120. doi: 10.1016/S0376-0421(02)00068-4

[50] Kessler, M., and Wagner, S. "Source-time dominant aeroacoustics," Computers \& Fluids Vol. 33, No. 5-6, 2004, pp. 791800.

doi: 10.1016/j.compfluid.2003.06.012

[51] Casalino, D. "An advanced time approach for acoustic analogy predictions," Journal of Sound and Vibration Vol. 261, No. 4, 2003, pp. 583-612.

doi: 10.1016/S0022-460X(02)00986-0

[52] Xu, C., Mao, Y., Hu, Z., and Gorbaniasl G. "Vector Aeroacoustics for a uniform mean flow: acoustic intensity and acoustic power," AIAA Journal, Submitted. 